\title{
An inside look at a biofilm: Pseudomonas aeruginosa flagella bio-tracking
}

Eden Ozer ${ }^{1, \dagger}$, Karin Yaniv ${ }^{3, \dagger}$, Einat Chetrit ${ }^{4}$, Anastasya Boyarski, Michael M. Meijler ${ }^{5,6}$, Ronen

$$
\text { Berkovich }^{2,4} \text {, Ariel Kushmaro }{ }^{2,3 *}, \text { Lital Alfonta }{ }^{1,2,5, *}
$$

$\dagger$ These authors contributed equally.

${ }^{1}$ Department of Life Sciences, Ben-Gurion University of the Negev, POBox 653, Beer-Sheva 8410501, Israel.

${ }^{2}$ Ilse Katz Institute for Nanoscale Science and Technology, Ben-Gurion University of the Negev, POBox 653, Beer-Sheva 8410501, Israel.

${ }^{3}$ Avram and Stella Goldstein-Goren Department of Biotechnology Engineering, Ben-Gurion University of the Negev, POBox 653, Beer-Sheva 8410501, Israel.

${ }^{4}$ Department of Chemical Engineering, Ben-Gurion University of the Negev, POBox 653, Beer-Sheva 8410501, Israel.

${ }^{5}$ Department of Chemistry, Ben-Gurion University of the Negev, POBox 653, Beer-Sheva 8410501, Israel.

${ }^{6}$ National Institute for Biotechnology in the Negev, Ben-Gurion University of the Negev, POBox 653, Beer-Sheva 8410501, Israel.

* Corresponding authors e-mails: arielkus@bgu.ac.il; alfontal@bgu.ac.i1 


\section{Abstract}

The opportunistic pathogen, Pseudomonas aeruginosa, a flagellated bacterium, is one of the top model organisms for studying biofilm formation. In order to elucidate the role of the bacteria flagella in biofilm formation, we developed a new tool for flagella bio-tracking. We have sitespecifically labeled the bacterial flagella by incorporating an unnatural amino acid into the flagella monomer via genetic code expansion. This enabled us to label and track the bacterial flagella during biofilm maturation. Direct, live imaging revealed for the first-time presence and synthesis of flagella throughout the biofilm lifecycle. To ascertain the possible role of the flagella in the strength of a biofilm we produced a "flagella knockout" strain and compared its biofilm to that of the wild type strain. Results showed a one order of magnitude stronger biofilm structure in the wild type in comparison to the flagella knockout strain. This suggests a newly discovered structural role for bacterial flagella in biofilm structure, possibly acting as a scaffold. Based on our findings we suggest a new model for biofilm maturation dynamic and underscore the importance of direct evidence from within the biofilm.

Keywords: Pseudomonas aeruginosa, Genetic code expansion, Flagella, Biofilm formation 


\section{Main}

Pseudomonas aeruginosa ( $P$. aeruginosa) is a well-studied opportunistic pathogen ${ }^{1}$. Despite the fact that quorum-sensing and biofilm formation have been studied for the past 40 years, new information is constantly being reported ${ }^{2,3}$. Biofilms provide a more resistant form of existence for bacteria than their planktonic forms, proffering them with protection from possible stressors. They therefore, have been intensely studied for their complexity and the mechanisms involved in their life cycle $e^{4,5}$. Indeed, any new information emerging from these studies is crucial, allowing the development of new and diverse strategies to resist infections.

The biofilm lifecycle is composed of several commonly reported steps ${ }^{1,6-8}$. Initially, planktonic bacteria propel themselves to a proximal surface, followed by an irreversible attachment to the surface. Once attachment is established, exo-polymeric substances are secreted from within the cells to generate a matrix of a supporting microenvironment for the dividing cells and to initiate formation of micro-colonies. Next, mushroom-like structures start to emerge. Finally, the cells secrete enzymes to digest the exo-polymeric substances at the top of the grown mushroom-like structures where the newly flagellated cells are released in a planktonic form to attach to new exposed surfaces.

The flagella, the bacterial rotor with its unique structure, is therefore an inseparable part of biofilm research ${ }^{9}$. Several reports have expressed a consensus regarding the importance of flagella in biofilm formation, specifically in its initiation ${ }^{10}$. However, despite a consensus among researchers that flagella are not present during biofilm maturation but only in the dispersion stage, reports regarding this are still somewhat constradicting ${ }^{10-16}$. Therefore, it is necessary to assess the presence and possible role of flagella in biofilms. Direct imaging of these organelles inside a developing biofilm may thus shed a better light on their role in biofilm formation and 
maintenance as well as provide an improved understanding of what occurs during the biofilm

25 lifecycle.

To date, live-cell imaging can be obtained through different approaches, however genetic code expansion, the reassignment of codons and incorporation of an unnatural amino acid (Uaa) into proteins ${ }^{17}$, displays advantages over other methodologies, and is gaining increasing exposure and momentum ${ }^{18-21}$. Genetic code expansion systems are being constantly improved, expanded and adapted to a growing number of organisms ${ }^{22-25}$. This technique aids in improving imaging. For example, the incorporation of a Uaa may alleviate the need for large and bulky labeling agents, such as fluorescent proteins or antibodies. Moreover, a protein with a site-specifically incorporated Uaa can be labeled using bio-orthogonal chemistry and serve as a specific reporter inside cells. Incorporating Uaa into $P$. aeruginosa flagellum enables live-cell imaging of flagella inside the complex environment of a biofilm. Herein, we present a robust and orthogonal genetic code expansion system in $P$. aeruginosa designed for flagella labeling for in-vivo flagella biotracking in a live and growing biofilm that revealed novel information regarding the biofilm lifecycle.

\section{Results}

Genetic code expansion of $\boldsymbol{P}$. aeruginosa. For a Uaa incorporation into $P$. aeruginosa proteins, a new plasmid was constructed (Fig. 1a). The plasmid harbors an orthogonal translation system (OTS), as well as a reporter gene for system validation. An OTS, composed of a tRNA and tRNA-synthetase pair, could be considered orthogonal if it does not interact with native translational components ${ }^{26}$. Methanosarcina mazei pyrrolysyl orthogonal translation system ${ }^{27}$ (MmPyl OTS) has been previously found to be orthogonal in several organisms, including gram- 
46 negative bacteria, and was therefore our choice. Combined with MmPyl OTS, a GFP reporter

47 gene encoded for Uaa incorporation.

The $P$. aeruginosa genetic code expansion plasmid (pPaGE) was assembled using $P$.

49 aeruginosa endogenous promoters and terminators. Our rationale was that the bacterium will

50 benefit from an attempt to maintain physiologically relevant expression levels of an exogenous

51 OTS. We thus identified the most abundant codon in $P$. aeruginosa PAO1 genome ${ }^{28}$, which was

52 found to be the CUG codon encoding for leucine (Leu). The native promoters and terminators of

53 Leu-tRNA and Leucil-tRNA-synthetase were assigned as the upstream and downstream regions

54 of Pyl-tRNA and Pyl-tRNA-synthetase respectively. Planning a future expression of flagella

55 protein, the $f l i C$ endogenous promoter for flagellin expression, was chosen to serve as a promoter

56 for the GFP reporter in the pPaGE plasmid. Toxicity tests for the Uaas: propargyl-L-lysine (PrK

57 (1)) and azido-carboxy-lysine (AzCK (2)) (Fig. 1b.1 and Fig. 1b.2, respectively) were performed 58 and have shown normal growth rates (Fig. S1). 

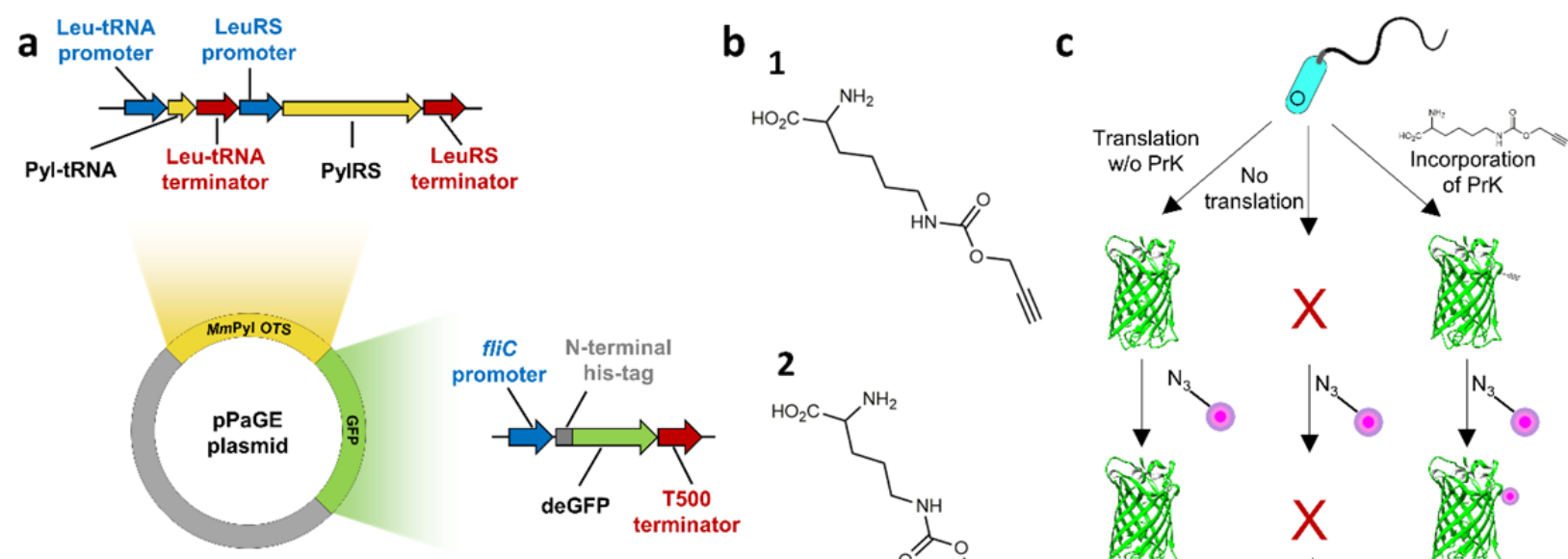

\section{2}

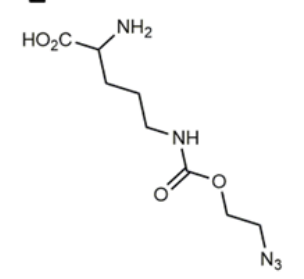

d

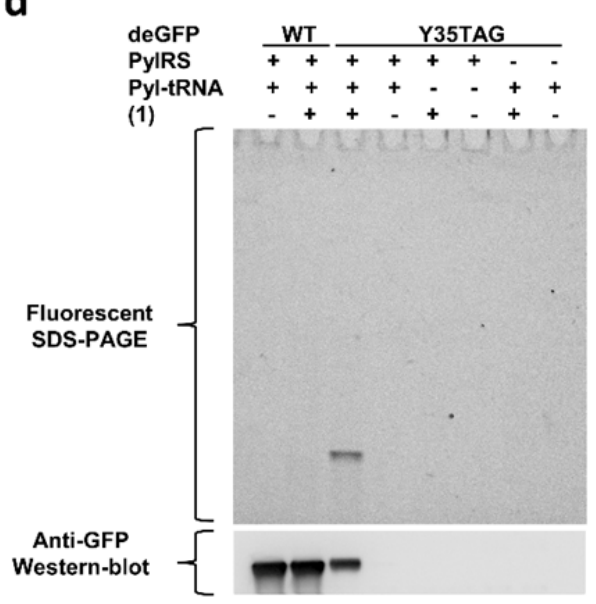

e

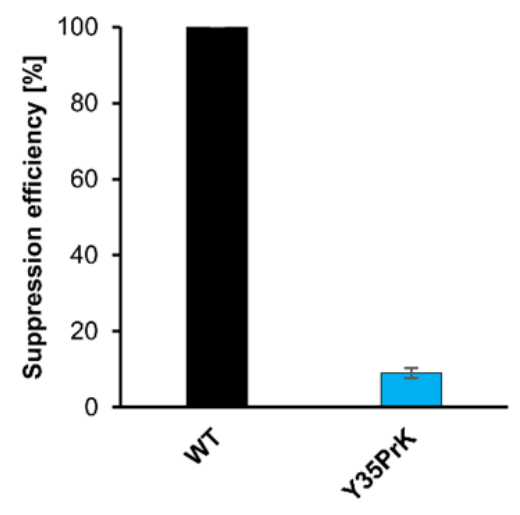

Fluorescent SDS-PAGE
+

Western-blot

f

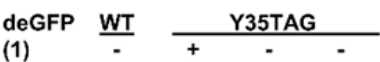

(2)

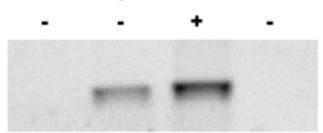

Figure 1| Genetic code expansion system of $\boldsymbol{P}$. aeruginosa using GFP. (a) Plasmid map of pPaGE. (b) Uaas used in this study, $\operatorname{PrK}(\mathbf{1})$ and $\mathrm{AzCK}$ (2). (c) Schematic representation of possible translation outcomes for midgene TAG mutants. (d) Lysed samples following a click reaction to an azide-containing fluorophore analyzed through fluorescent SDS-PAGE and anti-GFP Western-blot analyses. (e) Suppression efficiency of GFP Y35PrK mutant. (f) Lysed samples following a click reaction to either an azide or an alkyne containing fluorophore analyzed through fluorescent SDS-PAGE.

We reassigned the TAG stop-codon for site-specific incorporation of Uaas and introduced a TAG mutation to the GFP reporter gene at its $35^{\text {th }}$ site. MmPyl OTS orthogonality in P. aeruginosa was tested next. Several protein elongation scenarios for a premature TAG introduction to GFP were tested, as well as the possibility of non-specific incorporation of a Uaa into the host organism proteome (Fig. 1c, Fig. 1d). For that purpose, pPaGE variants containing partial OTSs were generated by the removal of Pyl-tRNA or Pyl-tRNA-synthetase (PylRS). Cells were grown, lysed and analyzed using fluorescent chemical conjugation and anti-GFP Western- 
75 blot analyses (Fig. 1d). In order to incorporate (1) into GFP, a click reaction using $\mathrm{Cu}(\mathrm{I})-$ 76 catalyzed azide-alkyne cycloaddition $(\mathrm{CuAAC})^{29}$ to an azide-bearing fluorophore was conducted.

77 Since there are no endogenous alkynes in bacteria, this experiment was meant to reveal whether

78 there exists any (1) that was misincorporated in response to the TAG stop-codon in the bacterial

79 proteome. Another possibility that needed to be ruled out was if PylRS can aminoacylate

80 endogenous tRNAs, where (1) would have been incorporated into random locations in the

81 genome. Both scenarios, resulting in non-specific fluorescent labeling into endogenous proteins.

82 On the other hand, if Pyl-tRNA is not orthogonal and is aminoacylated by the host organism's

83 tRNA-synthetases by natural amino acids, full-length GFP may still be synthesized and observed

84 in the Western-blot.

85 When examining all the protein expression options as seen in Figure 1d, we did not observe fluorescent labeling or GFP expression in the presence of the partial OTS variants. This

87 directly proved MmPyl OTS's orthogonality for the first time. Following the establishment that our system is indeed orthogonal, we were then able to analyze proper Uaa incorporation into GFP. Wild type (WT) GFP expression was observed either in the presence or absence of (1) and 90 could be seen only in the Western-blot analysis and not in the fluorescent gel. This indicated that 91 there was no incorporation of (1) into WT GFP. When the whole OTS was present together with 92 (1), a fully elongated GFP was detected in the Western-blot analysis. In addition, a clear 93 fluorescent labeling corresponding to GFP in size was visible. This not only indicated that the 94 reporter gene was able to be synthesized but also verified the presence of (1) inside the protein. 95 Indeed, when those cells were grown in the absence of (1), no expression was observed, 96 signifying that the expressed protein was not a result of a read-through event by any natural 97 amino acid. Final validation was performed using electrospray mass spectrometry (ESI-MS) 
(Fig. S2), where the deconvoluted mass corresponded to GFP molecular weight with the desired (1) at position 35 instead of a tyrosine in the WT protein. Thus, it was concluded that Uaa was successfully incorporated and encoded for, in a protein expressed in P. aeruginosa.

Suppression efficiency for Uaa incorporation into GFP was calculated as just under 10\% (Fig. 1e). While offering information on the incorporation efficiency, it is important to note the context effects $^{30}$ at play in this methodology which may alter incorporation yields as well as suppression efficiency. For example, having the $f l i C$ promoter may impair incorporation into GFP but may be better for Uaa incorporation into a flagellin. In order to further establish the generated system following genetic code expansion with (1), (2) was also incorporated into GFP to test system's flexibility, moreover (2) has the ability to undergo a copper-free click chemistry $^{29}$ which makes it useful for future in-vivo applications. Here, (2) was also detected through copper-catalyzed click chemistry, this time to an alkyne-containing fluorophore, and resulted in positive incorporation (Fig. 1f). Thus, genetic code expansion was successfully achieved and the system's orthogonality was established in $P$. aeruginosa, using two different Uaas.

Flagella "bio-tracking" in a biofilm. Next, we incorporated a Uaa into the endogenous gene of flagella. The flagellum is a complex machinery composed of several genes and proteins that generates filaments extending up to $15 \mu \mathrm{m}$ in length ${ }^{31,32}$. Although assembled by a consortium of proteins, the flagellin protein encoded by the $f l i C$ gene is the repeating monomer subunit giving the flagellum its high aspect ratio. We decided to introduce modifications mainly in the D3 domain of flagellin, facing outwards in the cylindrical structure of flagellum, thus minimizing possible interference with filament assembly ${ }^{32}$. We modulated the $P$. aeruginosa flagellum that is composed of 41 subunits (based on PDB accession number 5wk5) with (1) 
121 incorporated at flagellin's $248^{\text {th }}$ position (Fig. 2a). This illustrates our ability to label the micro-

122 structure by using a Uaa as a reactive bioorthogonal chemical handle, holding vast potential for

123 bio-labeling and other applications.

124 We reasoned that replacing the native flagellin copy into the genome of $P$. aeruginosa

125 with an incorporated Uaa may result in a short flagellum, leading to artifacts, as Uaa

126 incorporation is a slower process than native amino acids incorporation ${ }^{33}$. For that reason, we

127 chose an integrative approach: while encoding for a Uaa incorporation into the fliC gene

128 harbored in the $\mathrm{pPaGE}$ plasmid, we still retained native flagellin in the genome for a hybrid

129 assembly into a single unified flagellum. The integrated flagellum was thus predicted to have

130 fewer chemical anchor points for bio-labeling. However, despite not having every monomer

131 subunit carry a Uaa, this technique still allowed the micro-fiber to be labeled. Using the $f l i C$

132 promoter, the downstream sequence from the inserted $f l i C$ gene was also chosen as the native

133 flagellin terminator, hence the sequence was completely identical to the native genomic sequence

134 apart from the introduced premature TAG stop-codon mutation (Fig. 2b). The new plasmid was

135 assembled and served for flagella genetic code expansion from that point onwards. 

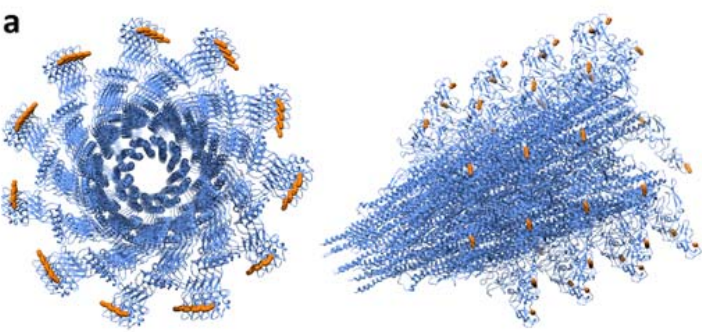

C

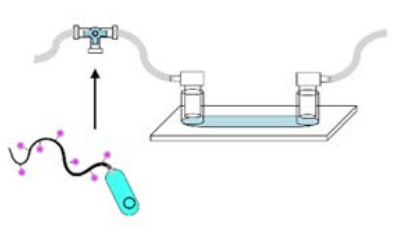

d
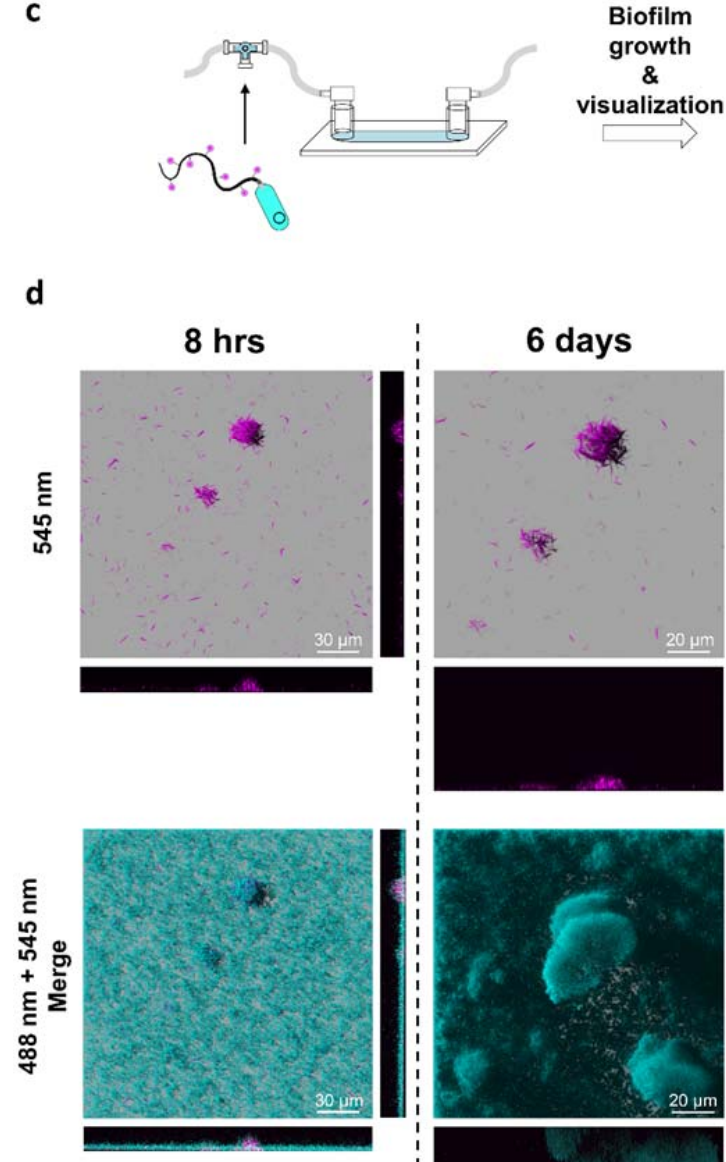

6 days
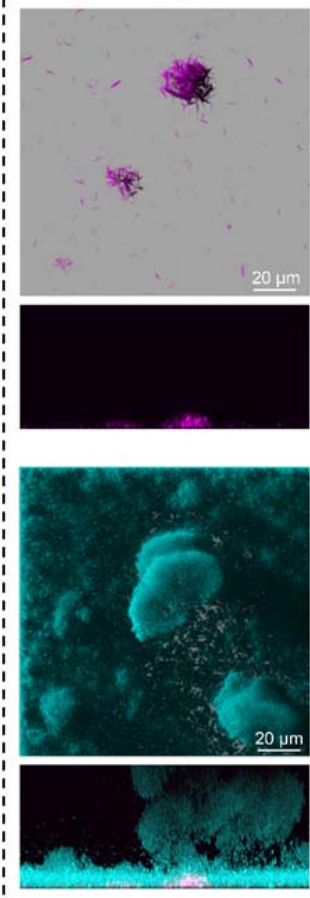

b
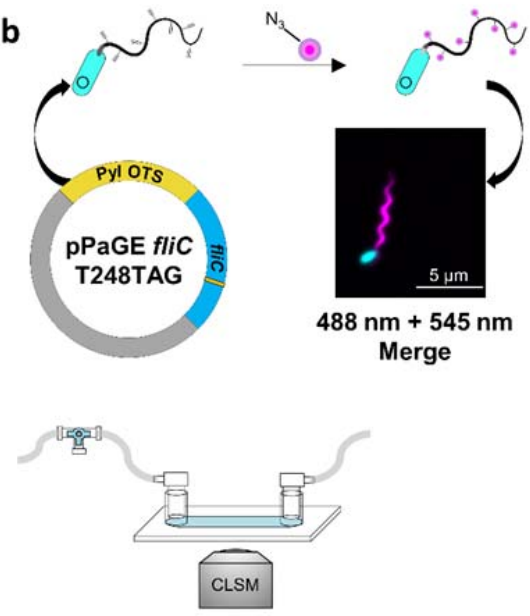

e

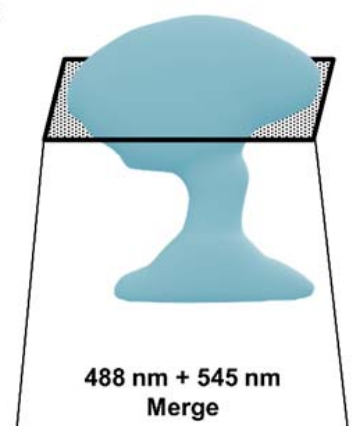

$31.5 / 59 \mu \mathrm{m}$
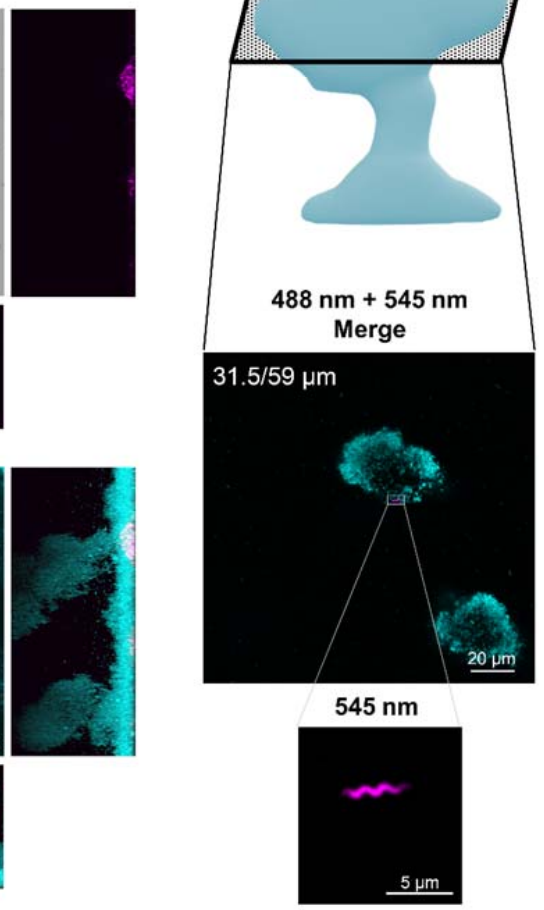

Figure 2| Flagellin Uaa incorporation and inoculated flagella survival in a biofilm. (a) Theoretical model of $P$. aeruginosa flagella filament with incorporated (1) at the D3 domain (based on PDB accession number: 5WK5) left: a top view; right: a side view, Uaa is an orange sphere. (b) Predicted system functionality and resulting fluorescent imaging using confocal laser scanning microscopy (CLSM). Flagella with an incorporated Uaa were labeled fluorescently (magenta) using PAO1 encoding genomic GFP reporter (gGFP) (cyan). Planktonic bacteria were imaged with incorporated (1) in the flagellum. (c) Schematic representation of the flow-cell and experimental setup for bio-tracking of flagella used for biofilm inoculation throughout biofilm maturation. Flagella with a Uaa were fluorescently labeled and used as inoculation cells for continuous biofilm growth for up to 6 days. Biofilm development and flagella were followed using CLSM. (d) Inoculated bacteria in a biofilm, pre-labeled prior to inoculation, monitored inside biofilm's 3D structure development using CLSM. (e) Pre-labeled flagella located in a mature biofilm at mid-height after 6 days of growth. Full scale and resolution images are available in the supplementary information (SI) file Figs. S6-S11. 
$\mathrm{pPaGE}$ fliC was transformed into $P$. aeruginosa and resulted in (1) incorporation into

150

151

152

153

154

155

156

157

158

159

160

161

162

163

164

165

166

167

168

169

170

171

flagellin monomers (Fig. S3). It was also established that exogenous flagellin expression does not affect flagellum synthesis in each individual cell (Fig. S4). Next, the system was used for live-cell imaging using a click reaction. pPaGE fliC was inserted into $P$. aeruginosa PAO1 gGFP strain, carrying a genomic GFP reporter gene for the convenience of whole-cell detection. Using a confocal laser scanning microscope (CLSM), successfully labeled flagella were observed including its unique wave-like morphology features indicating a repetitive occurrence of the incorporated label (Fig. 2b, magenta). No signal was observed when expression was attempted in the absence of (1) (Fig. S5). Despite the fact that not every monomer was labeled, the generated filament was successfully visualized, and the unique wave-like feature of flagella was recorded.

With this new labeling tool, it was now possible to "bio-track" flagella inside a biofilm. This technique enabled us to track the labeled inoculated flagella, attached to the cells that were used to initiate biofilm formation. Hence, the following experimental setup was pursued (Fig. 2c): $P$. aeruginosa expressing genomic GFP, harboring the pPaGE fliC plasmid were grown in the presence of (1). Planktonic bacteria containing (1) were fluorescently labeled by a click reaction and inserted into a flow-cell. Labeled bacteria were grown for up to 6 days, while inoculated flagella were monitored inside the biofilm every few hours. Labeled flagella, with their distinct morphology, were clearly observed after initial surface attachment and growth in the flow-cell either as singles or as clusters of different sizes (Fig. 2d). The emergence of flagella clusters was a notable observation that was not reported before.

Flagella bio-tracking for a time period of up to 6 days of biofilm growth, enabled us to provide evidence regarding possible changes in the state of the flagella in a biofilm with time. For example, we could determine whether inoculated flagella are maintained or degraded within 
172 the biofilm. We could demonstrate in a direct manner that despite continued biofilm growth to

173 heights of above $59 \mu \mathrm{m}$ in total, the majority of flagella remained in approximately at the bottom

174 of the biofilm in the first $13 \mu \mathrm{m}$ of the biofilm (Fig. 2d). While most flagella seemed to remain at

175 the bottom of the biofilm, rarely we observed inoculated flagella in higher sections of a grown

176 biofilm, found at around $31.5 \mu \mathrm{m}$ (Fig. 2e). That means that not only inoculated labeled flagella

177 are still present and are not metabolized within the time frame of the experiment but also that

178 occasionally, they might reach higher regions in the biofilm through an unknown mechanism.

179 The notion of flagella movement inside the biofilm has been previously speculated in the 180 literature ${ }^{13,14}$, however this is the first direct evidence for its occurrence. Indeed, the images

181 presented here (Fig. 2e) serve as the first direct evidence to this hypothesis. It is important to

182 note that due to imaging limitations, it is hard to determine if the observed flagella are still

183 attached to a cell or not. However, individual flagella that are observed in the biofilm's mid-

184 height were most likely positioned there due to a response to bacterial signaling. Hence, this has

185 led us to believe that most of the observed flagella are indeed attached to bacterial cells.

Flagella synthesis in a biofilm. After locating the labeled inoculated flagella in the biofilm,

187 we wanted to ascertain whether we could track newly synthesized flagella in the biofilm and if so, where are they located in the biofilm. The biofilm lifecycle is divided into several steps where planktonic cells transform and grow together into mushroom-like structures. Interestingly, throughout the biofilm's growth, flagella synthesis is halted and is only re-initiated during the

191 final step of dispersion from the mature biofilm ${ }^{5,7,8,34-36}$. To date, the most accepted concept of 192 flagella synthesis in a biofilm is that it occurs in a compartment located at the upper section of 193 the mushroom-like structure in a grown biofilm. Therefore, using our system, we set out to label 194 the compartments at the top of the mushroom-like structures in order to directly visualize flagella 
195 inside them for the first time. Pre-labeled cells were used for flow-cell inoculation as before, but

196 this time the media supply for the flow-cell contained (1) for continued Uaa incorporation inside

197 the growing biofilm for 2,4 or 6 days. Following growth, the biofilm was re-labeled using the

198 same fluorophore (Fig. 3a).

a

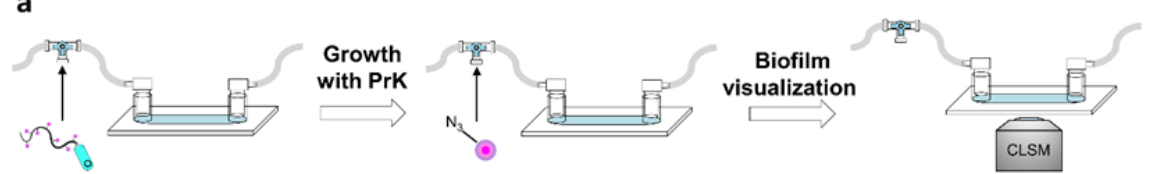

b

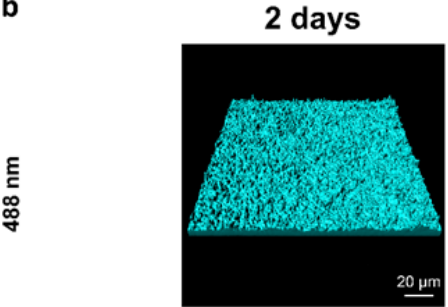

Whole biofilm

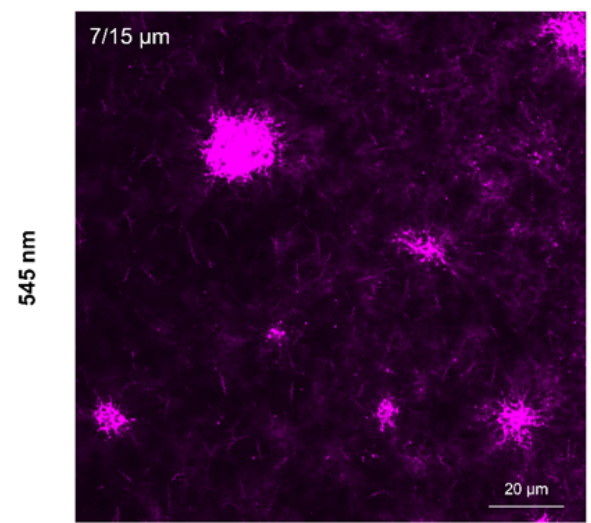

Mid-section

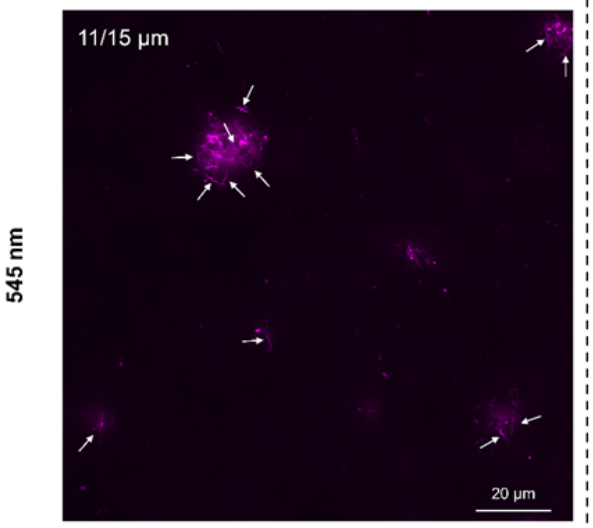

Upper-section
6 days

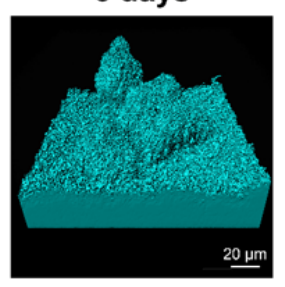

Whole biofilm

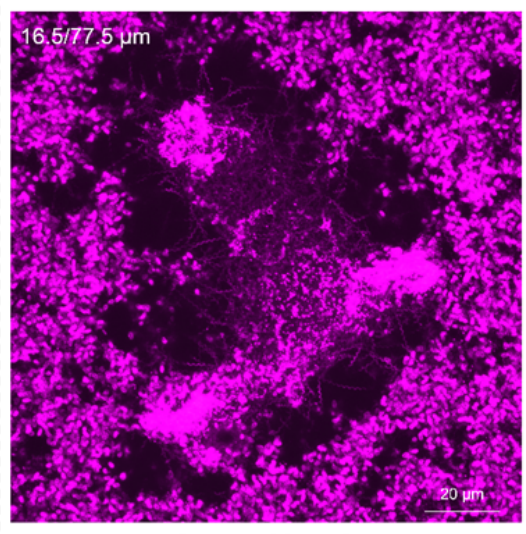

Lower mid-section

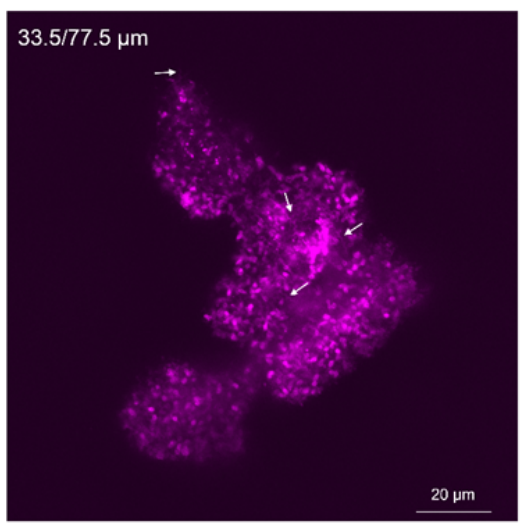

Mid-section 
Figure 3| Flagella synthesis during biofilm maturation. (a) Schematic representation of the experimental setup identifying flagella synthesis within the biofilm. Flagella with incorporated Uaa were fluorescently labeled and used as inoculation for continuous biofilm growth in the presence of Uaa for up to 6 days. Flagella were fluorescently labeled inside the biofilm at different biofilm's growth time points of 2 and 6 days and visualized using CLSM. (b) CLSM imaging from within the biofilm at different growth stages. For each time point during biofilm maturation, biofilm was imaged, and flagella were documented at different heights of the biofilm. Bacteria labeled in cyan and flagella in magenta. Enlarged images, as well as four-day old biofilm images are available in Figs. S12-S26.

Large quantities of fluorophore were trapped inside the viscous exo-polymeric matrix of the biofilm $^{6}$ and appeared as a bulk cloud-like signal. Flagella did not appear at the top of the mature biofilm, while many flagella were observed at the lower and middle sections of the biofilm throughout its growth (Fig. 3b, images of 4 days old biofilm and Figs. S17-S21). In the two-day old biofilm, flagella were located throughout the entire biofilm up to the height of $15 \mu \mathrm{m}$ on average. A four-day old biofilm also had flagella at the base of the biofilm as well at the height of approximately $22 \mu \mathrm{m}$ out of $30.5 \mu \mathrm{m}$ in total. Finally, a mature six-day old biofilm had abundant and noticeable flagella at mid-height $(33.5 \mu \mathrm{m})$ of the $77.5 \mu \mathrm{m}$ maximal height. These results not only confirmed our prediction of being able to detect flagella in the biofilm throughout its unique shape, but also contradicted the accepted consensus that flagella are located only at the top of mushroom-like structures in a mature biofilm.

Consistently seeing numerous flagella inside the biofilm throughout its whole lifecycle was unexpected. This is because according to the literature $5,7,8,34-36$, it appears that there is no flagella synthesis during biofilm growth. This notion was based mainly on indirect bulkpopulation analysis of DNA/RNA microarrays, total biomass calculations and proteomic analysis $^{15,37-39}$. This discrepancy provides important evidence for the importance of direct flagella visualization from within the biofilm, at an appropriate spatio-temporal resolution. When new flagella were observed from the biofilm's base up to approximately mid-height, we could only assume that previously indirect analysis had an averaging effect on the population. Another accepted aspect was that flagella exist at the top of micro-colonies in young biofilms, to allow 
229 flagella mediated movement in between micro-colonies ${ }^{13,14}$. A compartment containing flagella

230 at the top of a mushroom-like structure in a grown biofilm or any flagella in higher regions were

231 not observed even though they were easily detectable everywhere else in the biofilm.

232 Cells may detach from the mature biofilm by several ways; through erosion, bulk

233 detachment or by planktonic release ${ }^{8}$. In order to ascertain the possible changes occurring in

234 detaching cells, the Flow-cells' effluent was collected prior to whole biofilm labeling and was

235 labeled using a second fluorophore (with different excitation and emission wavelengths) to

236 distinguish between inoculated flagella and newly synthesized flagella (Fig. 4a). We collected

237 the biomass into chilled tubes immediately upon their exit from the flow-cells. Thus, we could

238 assume that any flagellum that was observed was synthesized inside the biofilm. Looking at the

239 double-labeled effluent of a young biofilm, new flagella were clear and abundant. This meant

240 that cells are frequently flagellated before leaving the biofilm (Fig. 4b). While correlating well

241 with known dispersion mechanisms from a mature biofilm, we expected to observe flagellated

242 cells leaving only the mature biofilm and were surprised by the continued flow of flagellated

243 cells in the effluent of our flow-cell set-up. (Fig. 4b, Figs. S27-S38). 


\section{a}

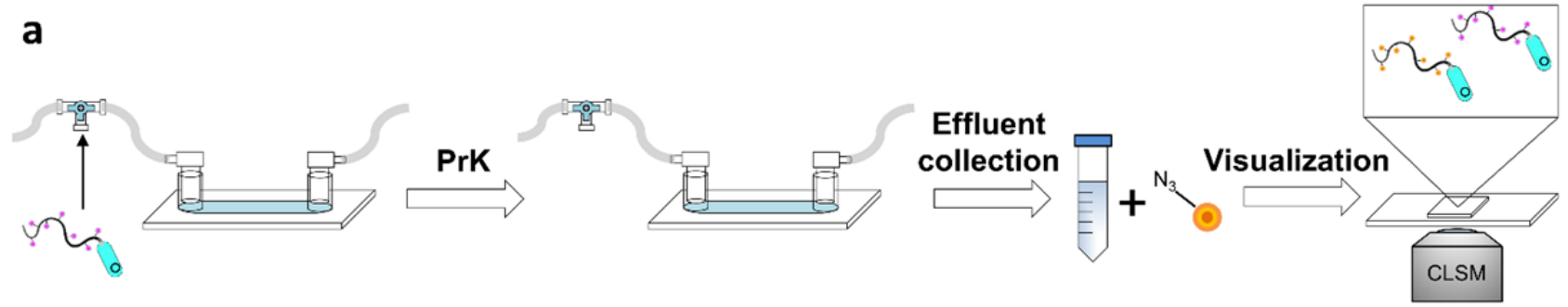

b

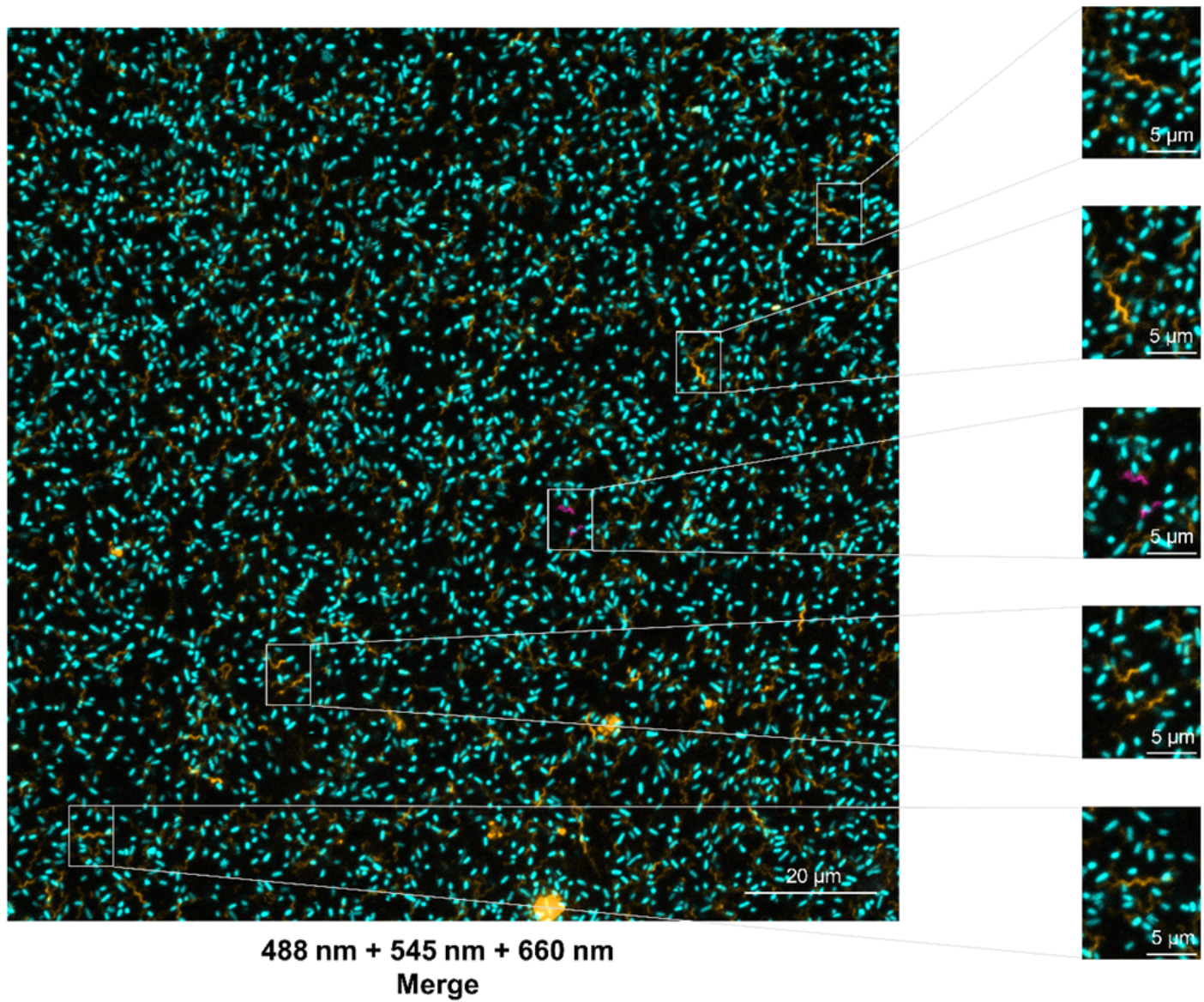

Figure 4| Dispersed flagellated cells. (a) Schematic representation of the experimental setup identifying dispersed cells from within the biofilm carrying synthesized flagella from before their release. Flagella with incorporated Uaa were fluorescently labeled and used as inoculation for continuous biofilm growth in the presence of Uaa for up to 6 days. Effluent was collected at different time points, labeled with a second, different fluorescent dye and visualized using CLSM. (b) CLSM imaging of dispersed flagellated cells from a two-day old biofilm. Bacteria labeled in cyan, inoculated flagella in magenta and new flagella in orange. Enlarged images, as well as dispersion images for 4-/6-day old biofilms, are available as Figs. S27-S38. 
256 reproducible. As mentioned previously, it is difficult to detect if the flagella that were observed

257 in the biofilm were connected to cells or not. Despite this, we showed that labeled inoculated

258 flagella were still attached to cells. This served as further support of our initial hypothesis,

259 suggesting most of the observed flagella were indeed still connected to a cell and that they

260 displayed some level of movement inside the biofilm.

261 Discovering continued flagellated dispersion is novel information regarding the biofilm

262 lifecycle model and reflects the observation that the biofilm is filled with flagella as was

263 discovered in this study. From a clinical aspect, mature biofilms are prone to planktonic

264 dispersion at times, causing exacerbations in chronic infections and afflicting new environment

265 within the host ${ }^{40}$. Usually, these exacerbations are in need of antibiotics treatment but are only

266 taken under consideration in case of a mature biofilm. Hence, continuous planktonic cells release

267 could affect the clinical view of possible treatments for chronic "biofilm infected" patients. All

268 the information gathered in this work, enabled us to portray an updated and more accurate model

269 for the biofilm lifecycle (Fig. 5). 


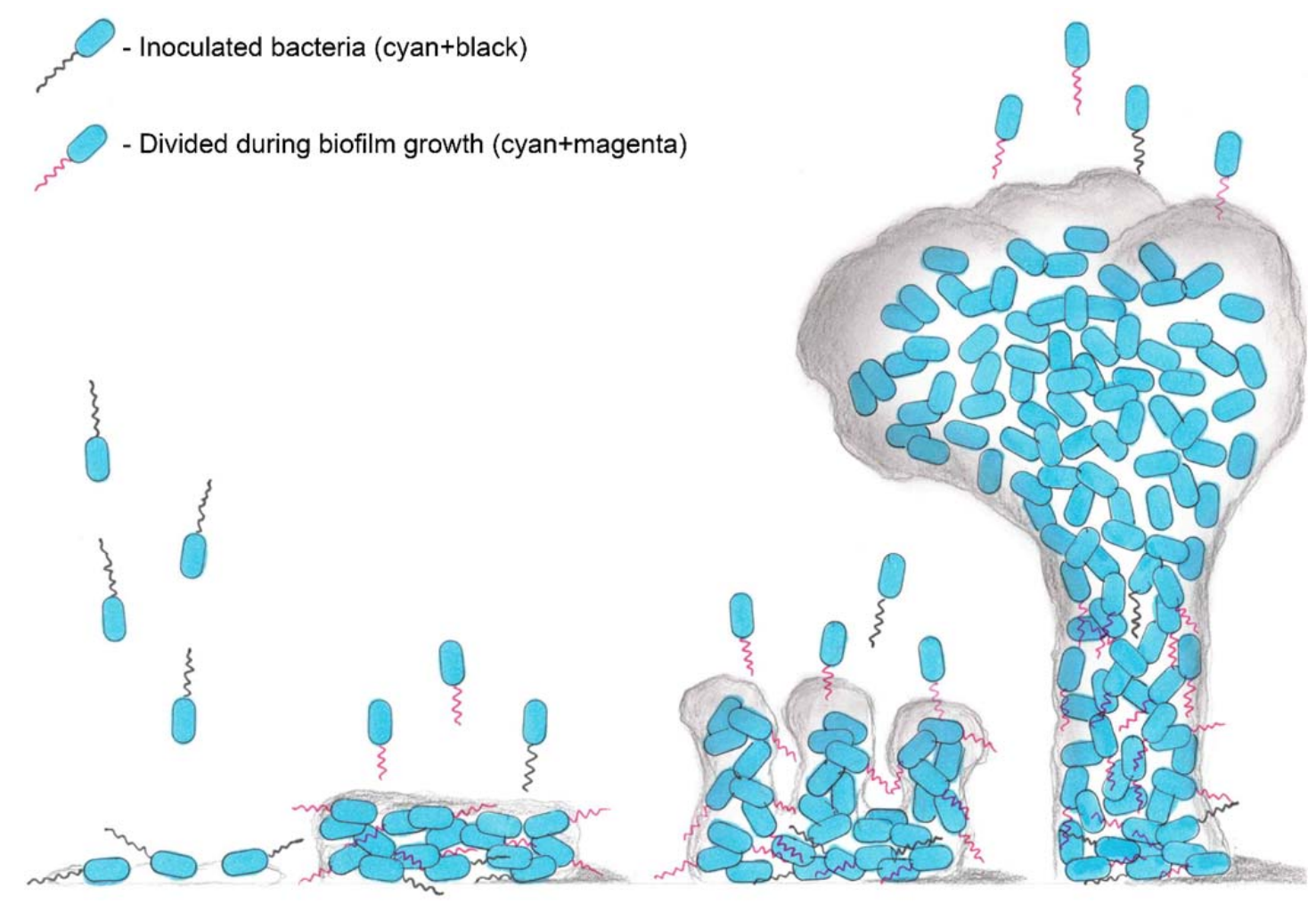

271 to why the flagella are formed in cells inside the biofilm. We observed a visible grid-like

278 appearance of the flagella in the lower part of the biofilm (Fig. 6a). In addition, we observed Figure 5| Updated biofilm lifecycle model based on the presented discoveries.

Flagella's structural importance in a biofilm. Learning that flagella are continuously being synthesized within a growing biofilm, we asked ourselves whether they might play a possible role in a biofilm mechanical structure. Considering that flagella synthesis is energetically costly ${ }^{41}$ and that cells within the biofilm are mainly non-motile, there must be a reasonable explanation as

279 several bacteria assembling on a single flagellum as shown in Figure 6b. These observations 280 seemed of importance as the structure resembled a structural scaffold that supports a substantial 281 architecture. The idea of the role of flagella as a mechanical support in a biofilm has been

282 previously suggested in E. coli macrocolony biofilms but has yet to be demonstrated ${ }^{42}$. An 283 additional observation to support this hypothesis is the observation of Geobacter sulfurreducens 
a

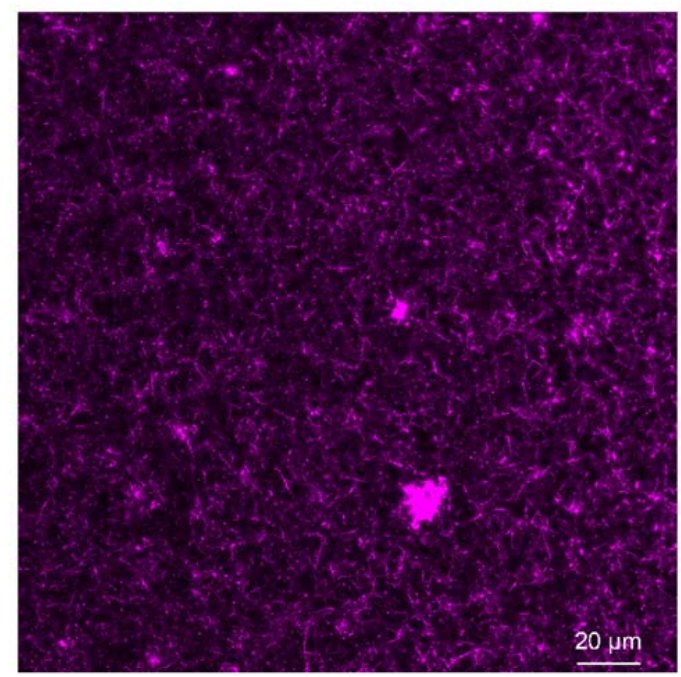

$545 \mathrm{~nm}$

\section{C}

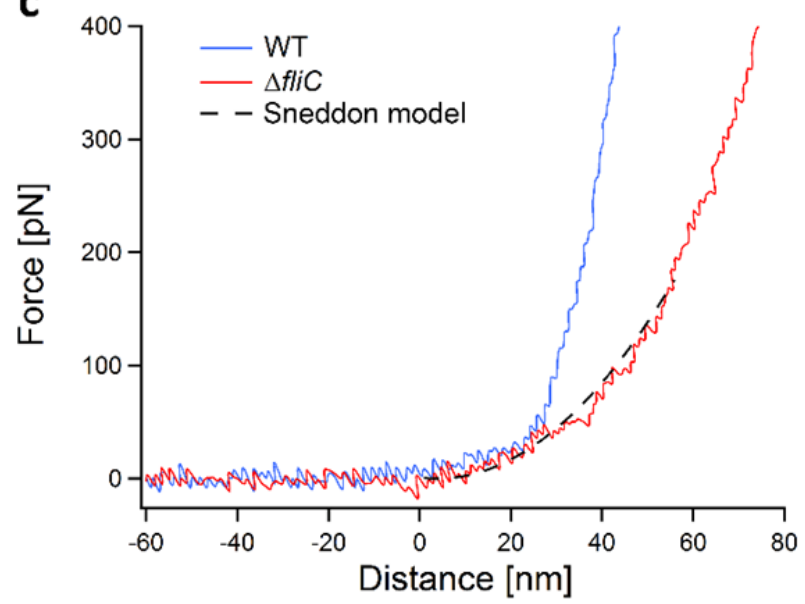

b

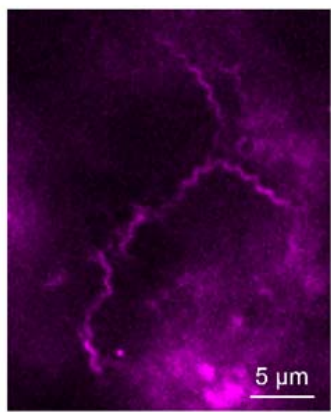

$545 \mathrm{~nm}$

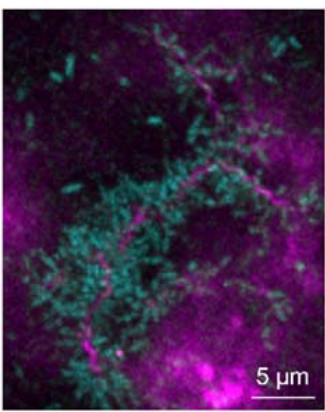

$488 \mathrm{~nm}+545 \mathrm{~nm}$ merge d

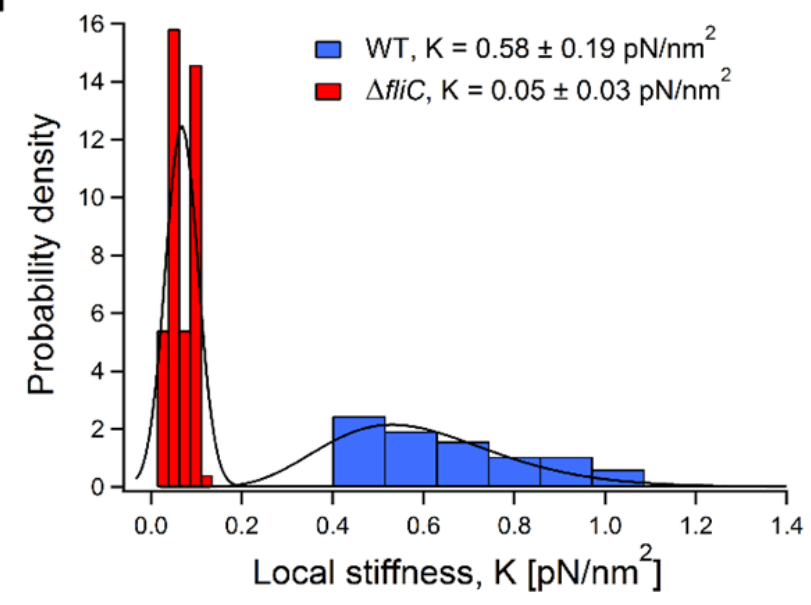

Figure 6| Flagella as physical support in a biofilm microenvironment. (a) CLSM imaging of the lower assembly on flagella in a growing biofilm. Bacteria labeled in cyan and flagella in magenta. Enlarged images are available as Figs. S39-S40. (c) Nanoindentation force-distance curves of the WT biofilm (blue) and a $\Delta$ fliC biofilm (red). The $\Delta f l i C$ curve is fitted with the Sneddon model (dashed black line), from which $\mathrm{K}$, the local stiffness modulus was estimated. (d) Local stiffness probability density distributions of WT biofilm (blue) and $\Delta f l i C$ biofilm (red), The distributions were fitted by relevant statistical models (black line), from which their means and standard deviations were calculated.

Using CRISPR Cas9 for genome editing, based on Streptococcus pyogenes Cas9 which was

298 adapted for $P$. aeruginosa genome editing ${ }^{43}$, we generated a sub-strain lacking the ability to 
299

300

301

302

303

304

305

306

307

308

309

310

311

312

313

314

315

316

317

318

319

320

321

produce flagella. This was carried out by deleting the flagellin gene $(\Delta f l i C)$ from the $P$. aeruginosa genome. Transmission electron microscope (TEM) imaging and the loss of its swarming abilities validated that the new strain does not express flagella (Fig. S41). We then attempted to identify differences between WT and $\Delta f l i C$ based biofilms. We hypothesized that mushroom-like structures will be affected by the absence of flagella. Despite this, we could not detect any distinct gross morphological differences between the two biofilms (Fig. S42). However, high-resolution scanning electron microscopy (HR-SEM) revealed noticeable visual difference between the two biofilms (Fig. S43). WT biofilm possessed fibers forming a web between the cells, while in the $\Delta f l i C$ biofilm cells were much more crowded and there were significantly less fibers. Since multiple string-like fibers were present in the biofilm and represented the complex environment surrounding the cells (sugars, proteins, DNA, etc.) it was not possible to use regular microscopic methods to determine morphologically which elements are flagella and which are not. Therefore, further comparison was performed using atomic force microscopy (AFM) on WT and $\triangle f l i C$ biofilms (Fig. S44). Though we did not find an indication in these images for the structural importance of the flagella to the biofilm, flagella were easily recognized within the WT biofilm. This serves as further validation of the consistent presence of flagella in the biofilm.

Hypothesizing that flagella contribute to the strength of the biofilm's structure, we decided to inspect the biofilm's mechanical strength and stiffness. A biofilm's mechanical strength is important to its survival. This characteristic is being actively studied in order to better understand the contribution of different elements to the exo-polymeric environment or to measure biofilm's resilience following different treatments ${ }^{44-46}$. For that purpose, we used AFM nanoindentation measurements on the two variants' mature biofilms. If the hypothesis is correct 
322 and the flagella take part in the biofilms' structural strength, then a biofilm lacking flagella

323 should be mechanically weaker. Figure 6c shows examples of WT and $\Delta f i C$ (marked as blue and

324 red, respectively) biofilm force-distance traces obtained from the nanoindentation measurements.

325 Each indentation curve demonstrates specific elastic response. There is a clear difference in the

326 elastic response curvatures for the two biofilms indicating lower local stiffness for the $\Delta$ fliC

327 biofilm. Fitting the curves with the Sneddon model allowed the estimation of local elastic

328 stiffness, $\mathrm{K}$, as illustrated for the $\Delta f l i C$ biofilm (Fig. 6c black dashed line).

We analyzed 100 force-distance curves collected for each of the biofilms, enabling the

estimation of local stiffness and verification that the biofilms did not undergo irreversible interestingly displayed two spreading behaviors. While the stiffness of the $\Delta f l i C$ displayed a narrow normal distribution, the stiffness of the WT biofilm showed a wide heavy tail distribution. This means that the stiffness values of the WT biofilm spread over a wider range compared to those of the $\Delta f l i C$ biofilm, with stiffness values that can get considerably high. For this reason, the $\triangle f l i C \mathrm{~K}$ probability distribution was fitted with a normal (Gaussian) distribution,

337 and the WT K was fitted with Gamma distribution, in order to properly assess their collective 338 values, i.e., means and standard deviations (Fig. 6d). Local stiffness value estimated for WT 339 biofilm was one order of magnitude higher than $\Delta f l i C$ biofilm's local stiffness. This proved that the $\Delta f l i C$ biofilm is weaker in terms of physical strength $\left(\mathrm{K}_{\mathrm{WT}}=0.58 \pm 0.19 \mathrm{pN} / \mathrm{nm}^{2}\right.$ vs. $\mathrm{K}_{\Delta f l i C}=$

$3410.05 \pm 0.03 \mathrm{pN} / \mathrm{nm}^{2}$ ). This result implied that, indeed, flagella take a crucial part in the biofilm 342 mechanical strength, much like scaffolds in a construction. 
345 due to various mutations in flagella synthesis involved genes ${ }^{47}$. It was found that cystic fibrosis

346 isolates occasionally develop differently, in order to evade the human immune system that

347 mainly targets bacterial flagella. Recently, Harrison et. al. ${ }^{47}$ revealed the connection between the

348 loss of flagellum and overexpression of exopolysaccharides in biofilms created from cystic

349 fibrosis isolates. The nanoindentation results presented here can elucidate further details on this

350 fascinating mechanism, as it is possible that bacteria produce more exopolysaccharides in the

351 attempt to compensate for the loss of flagella and its mechanical support. Also, if flagella indeed

352 contribute to the biofilm's strength, future biofilm treatment approaches may potentially target

353 flagella, to weaken the biofilm's structure and improve antibiotics penetration. Such a strategy

354 may then be followed by an effective antibiotic treatment that would otherwise be less efficient

355 in a strong and fully functional biofilm, and give way to future studies in clinical/environmental

356 biofilms.

357 Discussion

The field of biofilm studies is of great importance for basic microbiological understanding as well as in medical applications. Being studied intensely for over 40 years there are still many pieces missing in the complex biofilm puzzle. Despite the vast interest, part of the reason for this great mystery is its complexity and the challenges in its manipulation. For the most part, biofilm research is performed through bulk population analysis ${ }^{15,37-39}$, resulting in the possibility of overlooking details that seem to be minor. It is likely that these details are those that are actually the crucial details for understanding the true nature and dynamics of the biofilm population. Direct evidence of factors from within the biofilm provides an important tool to tackle this challenge. The work presented here has focused on spatial and temporal localization 367 of flagella in the biofilm, providing direct visualization for the first time. 
A designated genetic code expansion system was developed for direct flagella labeling, enabling its direct identification and characterization in a mature biofilm. Following orthogonality establishment for the first time, this system was utilized for bio-tracking flagella

371 inside biofilms. Direct imaging revealed inoculated flagella persistence during initial biofilm

372 growth and maturation. It also revealed inoculated flagellar movement in the biofilm.

373 Furthermore, as opposed to what was previously reported regarding flagella synthesis in a

374 biofilm, we demonstrated that flagella are present in the biofilm throughout its entire lifecycle.

375 While the presence of flagellated cells in compartments at the top of mushroom-like structures

376 found in a grown biofilm are a common concept for dispersion of cells, we did not detect flagella

377 any higher than the mid-section of these structures. Furthermore, we found that flagellated cells

378 constantly left the biofilm, either carrying newly synthesized flagella or on rare occasions

379 inoculated flagella. A combination of these findings led us to create an updated biofilm lifecycle

380 model that includes the dynamics of flagella within these biofilms.

The unexpected discoveries regarding the presence of flagella inside the biofilm, led to a

382 possible explanation for the reason behind this bacterial behavior with regards to the analysis of biofilm's physical strength. The detection of scaffold-like structures of flagella inside the biofilm resembled mechanical support needed for architectural buildings. This was strengthened when native biofilm displayed higher physical strength compared to a biofilm lacking flagella. Accordingly, we concluded that flagella play an important role in providing mechanical and 387 physical support of the biofilm. Further research though is needed to clarify the exact details behind this newly suggested role of flagella in a biofilm setting. We do recognize the existing obstacles at play, however, the work presented herein strongly emphasizes the need for 
thus serve as a window for new research venues. We posit that the new knowledge, afforded by this novel model, and approach that uses genetically code expanded strains as presented here will serve in tackling clinical/environmental biofilm research and envision multiple other new studies in regards to $P$. aeruginosa.

\section{Acknowledgments}

Prof. Ehud Banin is greatly acknowledged for useful discussions. We would like to thank the Ilse Katz Institute for Nanoscale Science and Technology Shred Resource Facility for their technical contribution in image acquisition with Zeiss LSM880 Airyscan (Dr. Uzi Hadad), Verios 460L Thermo Fisher Scientific HR-SEM (Einat Nativ-Roth), Helios G4 UC Thermo Fisher Scientific dual-beam HR-SEM (Nitzan Maman) and MFP-3D-Bio AFM (Juergen Jopp). Dr. Anna Bakhrat's assistance in genetic engineering is thankfully acknowledged. Yoni Ozer and Itay Algov's graphical assistance is thankfully acknowledged. Esti Kramarsky Winter is acknowledged for writing assistance. We thank the Kreitmann School for graduate students for a Ph.D. fellowship (E.O., A.B., K.Y.) and the Ben-Gurion University for a continued support of our research (L.A., A.K.).

\section{Contributions}

E.O. and K.Y. share equal contribution to this paper, E.O. conceived, performed and analyzed experimentations, established genetic code expansion system and authored the manuscript, K.Y. conceived, performed and analyzed all experimentations and co-authored the manuscript, E.C. performed nanoindentation experiments, A.B. synthesized unnatural amino acids used in this research, M.M.M., perceived and advised with P. Aeruginosa biofilm experiments, R.B. supervised, analyzed and wrote manuscript regarding AFM measurements, A.K. conceived experiments, supervised, provided research facilities and edited the manuscript, 

manuscript.

\section{References}

418 1. Lee, K. \& Yoon, S. S. Pseudomonas aeruginosa Biofilm, a Programmed Bacterial Life for 419 Fitness. J. Microbiol. Biotechnol. 27, 1053-1064 (2017).

420

421

422

423

424

425

426

427

428

429

430

431

432

433

434

435

436

437

438

439

440

441

442

443

2. Papenfort, K. \& Bassler, B. L. Quorum sensing signal-response systems in Gram-negative bacteria. Nat. Rev. Microbiol. 14, 576-588 (2016).

3. Høiby, N. A short history of microbial biofilms and biofilm infections. Apmis 125, 272275 (2017).

4. Parsek, M. R. \& Greenberg, E. P. Sociomicrobiology: The connections between quorum sensing and biofilms. Trends Microbiol. 13, 27-33 (2005).

5. Lappin-Scott, H., Burton, S. \& Stoodley, P. Revealing a world of biofilms-the pioneering research of Bill Costerton. Nat. Rev. Microbiol. 12, 781-787 (2014).

6. Maunders, E. \& Welch, M. Matrix exopolysaccharides; the sticky side of biofilm formation. FEMS Microbiol. Lett. 364, 1-10 (2017).

7. McDougald, D., Rice, S. A., Barraud, N., Steinberg, P. D. \& Kjelleberg, S. Should we stay or should we go: Mechanisms and ecological consequences for biofilm dispersal. Nat. Rev. Microbiol. 10, 39-50 (2012).

8. Petrova, O. E. \& Sauer, K. Escaping the biofilm in more than one way: Desorption, detachment or dispersion. Curr. Opin. Microbiol. 30, 67-78 (2016).

9. Barken, K. B. et al. Roles of type IV pili, flagellum-mediated motility and extracellular DNA in the formation of mature multicellular structures in Pseudomonas aeruginosa biofilms. Environ. Microbiol. 10, 2331-2343 (2008).

10. Belas, R. Biofilms, flagella, and mechanosensing of surfaces by bacteria. Trends Microbiol. 22, 517-527 (2014).

11. Blair, K. M., Turner, L., Winkelman, J. T., Berg, H. C. \& Kearns, D. B. A molecular clutch disables flagella in the Bacillus subtilis biofilm. Science (80-. ). 320, 1636-1638 (2008).

12. Liu, X. et al. Flagella act as Geobacter biofilm scaffolds to stabilize biofilm and facilitate 
extracellular electron transfer. Biosens. Bioelectron. 146, 111748 (2019).

445

446

447

448

449

450

451

452

453

454

455

456

457

458

459

460

461

462

463

464

465

466

467

468

469

470

471

472

473

474

13. Klausen, M. et al. Biofilm formation by Pseudomonas aeruginosa wild type, flagella and type IV pili mutants. Mol. Microbiol. 48, 1511-1524 (2003).

14. Tolker-Nielsen, T. et al. Development and dynamics of Pseudomonas sp. biofilms. $J$. Bacteriol. 182, 6482-6489 (2000).

15. Whiteley, M. et al. Gene expression in Pseudomonas aeruginosa biofilms. Nature 413, 860-864 (2001).

16. Claudine, B. \& Harwood, C. S. Cyclic diguanosine monophosphate represses bacterial flagella synthesis by interacting with the Walker a motif of the enhancer-binding protein FleQ. Proc. Natl. Acad. Sci. U. S. A. 110, 18478-18483 (2013).

17. Lemke, E. a. The exploding genetic code. ChemBioChem vol. 15 1691-1694 (2014).

18. Cohen, M., Ozer, E., Kushmaro, A. \& Alfonta, L. Cellular localization of cytochrome bd in cyanobacteria using genetic code expansion. Biotechnol. Bioeng. 117, 523-530 (2020).

19. Aloush, N. et al. Live Cell Imaging of Bioorthogonally Labelled Proteins Generated With a Single Pyrrolysine tRNA Gene. Sci. Rep. 8, 14527 (2018).

20. Kipper, K. et al. Application of Noncanonical Amino Acids for Protein Labeling in a Genomically Recoded Escherichia coli. ACS Synth. Biol. 6, 233-255 (2017).

21. Zhang, J. et al. Small Unnatural Amino Acid Carried Raman Tag for Molecular Imaging of Genetically Targeted Proteins. J. Phys. Chem. Lett. 9, 4679-4685 (2018).

22. Chemla, Y. et al. Expanding the Genetic Code of a Photoautotrophic Organism. Biochemistry 56, 2161-2165 (2017).

23. Gan, Q., Lehman, B. P., Bobik, T. A. \& Fan, C. Expanding the genetic code of Salmonella with non-canonical amino acids. Sci. Rep. 6, 39920-39926 (2016).

24. Bianco, A., Townsley, F. M., Greiss, S., Lang, K. \& Chin, J. W. Expanding the genetic code of Drosophila melanogaster. Nat. Chem. Biol. 8, 748-750 (2012).

25. Ernst, R. J. et al. Genetic code expansion in the mouse brain. Nat. Chem. Biol. 12, 776778 (2016).

26. Cervettini, D. et al. Rapid discovery and evolution of orthogonal aminoacyl-tRNA synthetase-tRNA pairs. Nat. Biotechnol. 38, 989-999 (2020).

27. Ozer, E. et al. In vitro suppression of two different stop codons. Biotechnol. Bioeng. 114, 1065-1073 (2017). 
475

476

477

478

479

480

481

482

483

484

485

486

487

488

489

490

491

492

493

494

495

496

497

498

499

500

501

502

503

504

505

28. Nakamura, Y. Codon usage tabulated from international DNA sequence databases: status for the year 2000. Nucleic Acids Res. 28, 292-292 (2000).

29. Singh, M. S., Chowdhury, S. \& Koley, S. Advances of azide-alkyne cycloaddition-click chemistry over the recent decade. Tetrahedron 72, 5257-5283 (2016).

30. Chemla, Y., Ozer, E., Algov, I. \& Alfonta, L. Context effects of genetic code expansion by stop codon suppression. Curr. Opin. Chem. Biol. 46, 146-155 (2018).

31. Chaban, B., Hughes, H. V. \& Beeby, M. The flagellum in bacterial pathogens: For motility and a whole lot more. Semin. Cell Dev. Biol. 46, 91-103 (2015).

32. Minamino, T. \& Namba, K. Self-assembly and type III protein export of the bacterial flagellum. J. Mol. Microbiol. Biotechnol. 7, 5-17 (2004).

33. Schlesinger, O. et al. Tuning of Recombinant Protein Expression in Escherichia coli by Manipulating Transcription, Translation Initiation Rates, and Incorporation of Noncanonical Amino Acids. ACS Synth. Biol. 6, 1076-1085 (2017).

34. Watnick, P. \& Kolter, R. Biofilm, City of Microbes. 182, 2675-2679 (2000).

35. Stoodley, P., Sauer, K., Davies, D. G. \& Costerton, J. W. Biofilms as Complex Differentiated Communities. Annu. Rev. Microbiol. 56, 187-209 (2002).

36. Sauer, K. et al. Pseudomonas aeruginosa Displays Multiple Phenotypes during Development as a Biofilm. J. Bacteriol. 184, 1140-1154 (2002).

37. Schniederberend, M. et al. Modulation of flagellar rotation in surfaceattached bacteria: A pathway for rapid surface-sensing after flagellar attachment. PLoS Pathog. 15, 1-30 (2019).

38. Morgan, R., Kohn, S., Hwang, S. H., Hassett, D. J. \& Sauer, K. BdlA, a chemotaxis regulator essential for biofilm dispersion in Pseudomonas aeruginosa. J. Bacteriol. 188, 7335-7343 (2006).

39. Tart, A. H., Wolfgang, M. C. \& Wozniak, D. J. The Alternative Sigma Factor AlgT Represses Pseudomonas aeruginosa Flagellum Biosynthesis by Inhibiting Expression of fleQ. J. Bacteriol. 187, 7955-7962 (2005).

40. Wu, H., Moser, C., Wang, H. Z., Høiby, N. \& Song, Z. J. Strategies for combating bacterial biofilm infections. Int. J. Oral Sci. 7, 1-7 (2015).

41. Zhao, K., Liu, M. \& Burgess, R. R. Adaptation in bacterial flagellar and motility systems: From regulon members to 'foraging'-like behavior in E. coli. Nucleic Acids Res. 35, 
506

507

508

509

510

511

512

513

514

515

516

517

518

519

520

521

522

4441-4452 (2007).

42. Serra, D. O., Richter, A. M., Klauck, G., Mika, F. \& Hengge, R. Microanatomy at Cellular Resolution and Spatial Order of Physiological Differentiation in a Bacterial Biofilm. MBio 4, 1-12 (2013).

43. Chen, W. et al. CRISPR/Cas9-based Genome Editing in Pseudomonas aeruginosa and Cytidine Deaminase-Mediated Base Editing in Pseudomonas Species. iScience 6, 222-231 (2018).

44. Tallawi, M., Opitz, M. \& Lieleg, O. Modulation of the mechanical properties of bacterial biofilms in response to environmental challenges. Biomater. Sci. 5, 887-900 (2017).

45. Baniasadi, M. et al. Nanoindentation of Pseudomonas aeruginosa bacterial biofilm using atomic force microscopy. Mater. Res. Express 1, 045411 (2014).

46. Zeng, G. et al. Functional bacterial amyloid increases Pseudomonas biofilm hydrophobicity and stiffness. Front. Microbiol. 6, 1-14 (2015).

47. Harrison, J. J. et al. Elevated exopolysaccharide levels in Pseudomonas aeruginosa flagellar mutants have implications for biofilm growth and chronic infections. PLOS Genet. 16, e1008848 (2020). 


\section{Online Methods}

\section{Reagents}

PrK ((S)-2-Amino-6-((prop-2-ynyloxy)carbonylamino)hexanoic acid) and AzCK ((S)-2-

526 Amino-6-((2-azidoethoxy)carbonylamino)hexanoic acid) were both synthesized according to a 527 protocol reported by Nguyen et al. $^{48}$. Tris(3-hydroxypropyltriazolylmethyl)amine (THPTA), 528 Tetramethylrhodamine-azide and Tetramethylrhodamine-alkyne were purchased from Sigma529 Aldrich (Rehovot, Israel). MB 660R Azide was a kind donation from Click Chemistry Tools

530 (Scottsdale, AZ, USA). All restriction enzymes were purchased from Thermo Fisher Scientific 531 (Waltham, MA, USA), while all DNA oligonucleotides were obtained from Syntezza Bioscience 532 (Jerusalem, Israel).

\section{Plasmids construction}

All plasmids for initial method establishment were constructed by a standard yeast assembly 535 protocol $^{22}$. The upstream and downstream regions from $P$. aeruginosa's leucyl translational system were chosen for OTS expression. Upstream and downstream regions of leucyl tRNA "flanked" pyrrolysyl tRNA, as well as for the upstream and downstream regions of leucyl tRNAsynthetase that "flanked" pyrrolysyl tRNA-synthetase. The deGFP reporter gene (a variant

540 also chosen to have a PAO1 endogenous promoter, the flagellin native promoter (fliC promoter).

541 It was chosen considering fliC gene was designed for Uaa incorporation after deGFP.

542 The initial construct (pPaGE Pyl TAG fliC prom deGFP WT NHis) was assembled in two 543 stages. First, pMRP9-1 backbone was amplified using primers 1 and 2 (table S1), without the 544 deGFP gene, and was assembled with the $2 \mu$ origin and URA3 selectivity gene for yeast.

545 Second, the new vector after restriction with BamHI was assembled with seven other PCR 
amplified parts, containing the OTS, deGFP expression gene and necessary promoters and terminators (primers 3-16, table S1). Endogenous regions of $P$. aeruginosa were amplified from PAO1 genome. N-terminal his-tag deGFP and its T500 terminator were amplified from the pBEST plasmid ${ }^{27}$. Pyrrolysyl tRNA-synthetase was amplified from pEVOL-Pyl plasmid described in previous work $^{27}$. Pyrrolysyl tRNA was assembled through primers 4 and 5 homology (table S1) during the yeast assembly. The pPaGE Pyl TAG fliC prom deGFP Y35TAG NHis construct was assembled in the same manner, only with a mutant deGFP amplified from the pBEST plasmid.

Variant for orthogonality testing of Pyl tRNA was generated through standard DNA collapse using HindIII. HindIII restriction, followed by self-ligation of the plasmid without the PylRS gene. During the initial construct generation, a deletion construct without Pyl tRNA was also created. This variant was used for orthogonality testing of PylRS.

A construct containing the $f l i C$ gene with a TAG mutation (pPaGE Pyl TAG fliC T248TAG) was assembled through Gibson assembly. pPaGE was restricted with NcoI for vector generation without the deGFP gene and T500 terminator. fliC gene had the TAG mutation installation as part of the assembly, by two pieces amplification. The gene, together with its downstream sequence, was amplified from the PAO1 genome using primers $17+20$ and 19+18 (table S1).

All plasmids inserted into E. coli underwent standard heat-shock transformation protocol.

All plasmids inserted into P. aeruginosa underwent standard electroporation protocol.

\section{Viability assay}

Bacterial liquid culture in LB-Miller, after $24 \mathrm{hrs}$ of growth at $37^{\circ} \mathrm{C}$, was diluted $1: 100$. The diluted culture was placed at $37^{\circ} \mathrm{C}$. Every $1 \mathrm{hr}$, a duplicate was measured for OD at a wavelength of $600 \mathrm{~nm}$ using a Synergy HT plate reader (Biotek, Winooski, VT, USA). After 17 hrs of 
569

570

571

572

573

574

575

576

577

578

579

580

581

582

measurements, when the cells reached growth plateau, the culture was left for incubation for another $10 \mathrm{hrs,}$ when a final measurement was taken. When needed, the culture was supplemented with final concentration of $1 \mathrm{mM}$ Uaa (optimal concentration found is shown in Fig S36). In case of plasmid containing bacteria, growth medium was supplemented with 300 $\mu \mathrm{g} / \mathrm{mL}$ carbenicillin.

\section{Suppression efficiency}

Five separate sets of liquid cultures were grown at $37^{\circ} \mathrm{C}$ for $24 \mathrm{hrs}$. Each set was tested for GFP expression and was composed of a native strain of $P$. aeruginosa, WT GFP and Y35TAG GFP with PrK. Following growth, each sample, from each set, was tested in triplicates for OD at a wavelength of $600 \mathrm{~nm}$ and GFP fluorescence by using a Synergy HT plate reader (Biotek, Winooski, VT, USA). Each sample's fluorescence was divided by the $\mathrm{OD}_{[600]}$ value. Values of WT and Y35TAG with PrK were normalized to the native strain's value of fluorescence/OD ${ }_{[600]}$. Finally, the suppression efficiency of each set was determined through value of normalized fluorescence $\backslash O D_{[600]}$ of mutant divided by the value of normalized fluorescence $\backslash \mathrm{OD}_{[600]}$ of WT.

Error bar in figure 1e could only be calculated for mutant expression (as WT is always 100\% by definition), representing the standard deviation between 5 different suppression efficiencies values calculated.

\section{Protein expression in PAO1 and cells lysis}

Culture growth: PAO1 harboring pPaGE variants, were grown in LB-Miller with $300 \mu \mathrm{g} / \mathrm{mL}$ carbenicillin at $37^{\circ} \mathrm{C}$ for $24 \mathrm{hrs}$. After growth, the cultures were diluted 1:100 for another $24 \mathrm{hrs}$ of growth. In case of needed Uaa addition, the culture was supplemented with final concentration of $1 \mathrm{mM}$ Uaa. 
Lysis: Following $24 \mathrm{hrs}$ of growth, $1 \mathrm{~mL}$ of culture was sedimented and resuspended with 1

594 10X protein extraction reagent (Merck, Billerica, MA, USA), Turbonuclease (Sigma, St. Louis, 595 MO, USA), Lysozyme (Sigma-Aldrich, Rehovot, Israel) and protease inhibitor (Merck,

596 Darmstadt, Germany). Cells were incubated with the lysis solution for 30 min at room 597 temperature while shaking, followed by $4^{\circ} \mathrm{C}$ centrifugation at $10000 \mathrm{~g}$ for $10 \mathrm{~min}$. Supernatant 598 containing soluble proteins fraction was taken for further analysis. ascorbate and $\mathrm{CuCl}_{2}$ were added to final concentration of $1.2 \mathrm{mM}, 2.5 \mathrm{mM}$ and $200 \mu \mathrm{M}$, respectively. A volume of $20 \mu \mathrm{L}$ of cell lysate was added to the reaction, followed by $1 \mathrm{hr}$ incubation at room temperature with shaking. Clicked samples were examined through SDS-

606 PAGE 4-20\% Expressplus protein gel (GenScript, Nanjing, China). Fluorescent SDS-PAGE 607 images were obtained through ImageQuant LAS 4000 imager (Fujifilm, Tokyo, Japan), using 608 green light (520 nm Epi) and a $575 \mathrm{~nm} \mathrm{Cy3} \mathrm{detection} \mathrm{filter.} \mathrm{Next,} \mathrm{when} \mathrm{Western-blot} \mathrm{was}$ 609 needed, SDS-PAGE was transferred to a membrane (Bio-Rad, Hercules, CA, USA) through

610 eBlot ${ }^{\circledR}$ protein transfer system (GenScript, Nanjing, China). Using goat T-19 anti GFP 611 antibody $^{49}$ (sc-5384, Santa Cruz, CA, USA) as a primary antibody and donkey anti goat IgG$612 \mathrm{HRP}^{50}$ (sc-2020, Santa Cruz, CA, USA) as a secondary antibody, standard Western-blot protocol 
613 was performed. Chemiluminescence imaging was done using ImageQuant LAS 4000 imager

614 (Fujifilm, Tokyo, Japan).

615

616

\section{Protein purification and mass spectrometry}

617

A $200 \mathrm{~mL}$ culture of the P. aeruginosa GFP Y35TAG mutant, in the presence of PrK, was

618 IMAC (Novagene, Madison, WI, USA) according to manufacturer guidelines. Elution fraction

619 was concentrated using Vivaspin 6, 10000 MWCO PES (Sartorius, Goettingen, Germany) and

620 concentrated fraction was analyzed by liquid chromatography mass spectrometry (LCMS)

621 (Finnigan Surveyor Autosampler Plus/LCQ Fleet, Thermo Scientific, Waltham, MA, USA).

622

Theoretical model of P. aeruginosa flagella

623

A monomer model of $P$. aeruginosa flagellin was obtained through SWISS-MODEL ${ }^{51}$ and a

624 PrK residue was incorporated in position 248 (PyMOL Molecular Graphics System, Version 2.0

625 Schrödinger, LLC). PDB file 5WK5, containing 41 subunits of the $P$. aeruginosa's filament core

626 (no outer protein structure of D2 and/or D3), was used as alignment reference for 41 SWISS-

627 MODEL generated monomers with PrK (PyMOL Molecular Graphics System, Version 2.0

628 Schrödinger, LLC). The final product is a theoretical model of a flagella filament, consisting of

62941 P. aeruginosa flagellin proteins, all of which contain PrK in the $248^{\text {th }}$ position.

630

631

632

633

634

635

\section{Live-cell click reaction and flow-cells construction}

$\mathrm{pPaGE}$ harboring $f l i C$ with TAG mutation at the $248^{\text {th }}$ site, was electroporated into PAO1 gGFP strain (a strain containing GFP expression gene in the genome, gGFP = genome GFP). Liquid culture in the presence of PrK, was grown at $37^{\circ} \mathrm{C}$ for $24 \mathrm{hrs}$. Grown culture at the volume of $200 \mu \mathrm{L}$ was centrifuged and resuspended with $20 \mu \mathrm{L}$ phosphate buffer $100 \mathrm{mM} \mathrm{pH} 7$. Click reagents were added to a final reaction volume of $50 \mu \mathrm{L}$. Azide-containing 545 
636

637

638

639

640

641

642

643

644

645

646

647

648

649

650

651

652

653

654

655

656

657

658

fluorophore was added to a concentration of $50 \mu \mathrm{M}$, while THPTA, sodium ascorbate and $\mathrm{CuCl}_{2}$ were added to a final concentration of $1.2 \mathrm{mM}, 2.5 \mathrm{mM}$ and $200 \mu \mathrm{M}$, respectively. Following 40 min incubation at room temperature with shaking, cells were washed three times with $1 \mathrm{~mL}$ of phosphate buffer $100 \mathrm{mM} \mathrm{pH} 7$ and were brought to a value of $\mathrm{OD}_{600}$ of 0.1 . A flow-cell system was constructed as described before ${ }^{52}$, using the labeled bacteria as inoculation for growth. Following $1 \mathrm{hr}$ of static attachment in the flow-cell at $30^{\circ} \mathrm{C}, \mathrm{AB}$ minimal growth media at temperature of $37^{\circ} \mathrm{C}$ was supplied at a rate of $4 \mathrm{~mL} / \mathrm{hr}$. Flow-cells were grown for up to 6 days while imaging for different experimental procedures was done using confocal laser scanning microscopy (CLSM).

For pre-inoculation labeled flagella tracking -flow-cell was constructed as described and was imaged using CLSM at 2 different channels (488 at cyan for bacteria and 545 at magenta for preinoculation labeled flagella) at specific locations every several hours for up to 6 days.

For effluent flagella tracking -flow-cell was constructed as described while AB minimal media was supplemented with $\operatorname{PrK}$ for continuous incorporation. Effluent of $\sim 15 \mathrm{~mL}$ was collected into ice following 2/4/6 days of growth. Effluent total volume was pelleted down and resuspended with $20 \mu \mathrm{L}$ phosphate buffer $100 \mathrm{mM} \mathrm{pH} \mathrm{7.} \mathrm{Click} \mathrm{reagents} \mathrm{were} \mathrm{added} \mathrm{to} \mathrm{a} \mathrm{final}$ reaction volume of $50 \mu \mathrm{L}$. Azide-containing 660 fluorophore was added to a concentration of 50 $\mu \mathrm{M}$, while THPTA, sodium ascorbate and $\mathrm{CuCl}_{2}$ were added to a final concentration of $1.2 \mathrm{mM}$, $2.5 \mathrm{mM}$ and $200 \mu \mathrm{M}$, respectively. Following $40 \mathrm{~min}$ incubation at room temperature with shaking, cells were washed two times with $1 \mathrm{~mL}$ of phosphate buffer $100 \mathrm{mM} \mathrm{pH} 7$ and were finally resuspended with $\sim 50 \mu \mathrm{L}$ PB $100 \mathrm{mM}$ pH 7. Labeled effluent (5 $\mu \mathrm{L}$ onto a glass slide) was imaged using CLSM at 3 different channels (488 at cyan for bacteria, 545 at magenta for pre-inoculation labeled flagella, 660 at orange for newly synthesized labeled flagella). 
For newly synthesized labeled flagella tracking within a biofilm - flow-cell was constructed

660

661

662

663

664

665

666

667

668

669

670

671

672

673

674

675

676

677

678

679

680

681

as described and grown for 2/4/6 days while $\mathrm{AB}$ minimal media was supplemented with $\operatorname{PrK}$ for continuous incorporation. Click reagents solution was prepared for a final reaction volume of $500 \mu \mathrm{L}$. Azide-containing 545 fluorophore was added to a concentration of $50 \mu \mathrm{M}$, while THPTA, sodium ascorbate and $\mathrm{CuCl}_{2}$ were added to a final concentration of $1.2 \mathrm{mM}, 2.5 \mathrm{mM}$ and $200 \mu \mathrm{M}$, respectively. The click mixture was slowly injected into the flow-cell chamber and remained stationary for $20 \mathrm{~min}$ at room temperature, followed by intensive wash with growth media at a rate of $16 \mathrm{~mL} / \mathrm{hr}$ at room temperature. Flow-cell was imaged using CLSM at 2 different channels (488 at cyan for bacteria, 545 at magenta for labeled flagella).

All mentioned experimental procedures were repeated for a minimum number of 5 different flow-cells.

\section{Confocal microscope settings and image analysis}

Confocal images were acquired using Zeiss LSM880 system (Zena, Germany). PlanApochromat 63x/1.4 Oil DIC M27 or Plan-Apochromat 40x/1.3 Oil DIC M27 objective were used. Cyan channel was imaged using $488 \mathrm{~nm}$ Argon laser (usually in the range of 5-15\%) with emission filter BP 493-556. Magenta channel was imaged using $561 \mathrm{~nm}$ DPSS laser (usually in the range of 2-4\%) with emission filter BP 570-624. Orange channel was imaged using $633 \mathrm{~nm}$ HeNe laser (usually in the range of 8-15\%) with emission filter BP 638-755.

Scanning resolution for all flow-cells was 1024x1024. Scanning resolution for effluent planktonic cells was $2048 \times 2048$.

Image analysis was performed using either ImageJ software (National institutes of health, USA) or IMARIS software (Bitplane AG, Zurich, Switzerland). Despite channel modifications in image analysis, a gamma value of 1.00 was strictly conserved in all images and analysis. 
Figure $2 \mathrm{~b}$ - all $9 \mathrm{Z}$ plains were stacked and channels were merged $(488+545)$ and a smooth

683

684

685

686

687

688

689

690

691

692

693

694

695

696

697

698

699

700

701

702

703

704

filter was used. (ImageJ)

Figures $2 d-3 D$ digital visualization of 545 alone or merged channels $(488+545)$ were at 6000x6000 dimensions and 900 dpi using “shadow” function. (IMARIS)

Figure $2 \mathrm{e}-$ Out of $118 \mathrm{Z}$ plains, 61-65 were stacked and 488+545 channels were merged.

(ImageJ)

Figures 3b -Digital bio-volume was saved as 6000x6000 and 900 dpi. 2 days mid-section was stacked from 13-16 Z plains. Upper-section was stacked from 20-25 Z plains. Lower midsection was stacked from 27-39 Z plains. Mid-section was stacked from 66-68 Z plains. (IMARIS)

Figures $4 \mathrm{~b}$ - Out of $13 \mathrm{Z}$ plains, image was stacked using 4-9 Z plains and saved as individual channels (488/545/660) or a merged image of all channels. (ImageJ)

Figure $6 \mathrm{a}-$ The $14 \mathrm{Z}$ plain was presented only in the 545 channel. (IMARIS)

Figure $6 b-Z$ plains of 23-26 were stacked and saved as individual 545 channel or a merged image of $488+545$. (IMARIS)

\section{P. aeruginosa PAO1 gGFP $\triangle$ fliC strain generation}

For the creation of a KO strain to flagella filament, a CRISPR/Cas9-based platform adapted to $P$. aeruginosa ${ }^{43}$ was utilized. Out of a 2-plasmids system, pCasPA plasmid was used as is, while a relevant pACRISPR plasmid had to be constructed using Gibson assembly to contain gRNA and complementary homology region to the genome. The gRNA used was chosen based on $\mathrm{CHOPCHOP}^{53}$ and complemented sites $168-174$ in fliC gene. Homology region was chosen as $500 \mathrm{bp}$ upstream to the ATG codon of $f l i C, 30 \mathrm{bp}$ from the end of the $f l i C$ gene (including stop-codon) and $500 \mathrm{bp}$ downstream to the stop-codon of $f l i C$, meaning deletion of $1437 \mathrm{bp}$ out 
of the $f l i C$ gene. Gibson assembly for pACRISPR with relevant gRNA and homology was done using primers 21-28 (table S1), following standard assembly and plasmid sequence verification. pCasPA was electroporated into PAO1 gGFP strain using standard protocol and grown on tetracycline $100 \mu \mathrm{g} / \mathrm{mL}$ selective plate. After first plasmid insertion, PAO1 gGFP pCasPA was grown over night at $37^{\circ} \mathrm{C}$, and was added with L-arabinose to final concentration of $2 \mathrm{mg} / \mathrm{mL}$ for 2 hrs incubation at $37^{\circ} \mathrm{C}$ (targeted for Cas 9 inductive protein expression). Cells then were prepared for electroporation using standard protocol. pACRISPR targeted for fliC deletion was electroporated into PAO1 gGFP pCasPA cells prepared in advance and grown on tetracycline $100 \mu \mathrm{g} / \mathrm{mL}+$ carbenicillin $150 \mu \mathrm{g} / \mathrm{mL}$. Colonies were screened for genome segment modification and chosen colonies were cured from both plasmids by plating on sucrose 5\% plates. Chosen colony was isolated, tested for positive plasmid curing, and tested by PCR for positive deletion (primers 29+30, table S1). PCR segment was purified using nucleospin gel and PCR clean-up (MACHEREY-NAGEL, Germany) and underwent Sanger sequencing with primer 31 (table S1).

\section{WT/KO strains flow-cells construction}

WT (PAO1 gGFP) or KO (PAO1 gGFP $\Delta f l i C)$ strain stationary grown liquid culture was diluted to $\mathrm{OD}_{600}$ of 0.1 and cells were inoculated in a flow-cell as described before. After $1 \mathrm{hr}$ of attachment at $30^{\circ} \mathrm{C}, 37^{\circ} \mathrm{C}$ heated $\mathrm{AB}$ minimal media at a rate of $4 \mathrm{~mL} / \mathrm{hr}$ was supplemented for 5 days of growth. Flow-cells were examined for biofilm structure and 3D maturation using CLSM.

\section{Transmission electron microscopy (TEM)}

Grown bacteria cultures were diluted 1:20. A carbon type-B TEM grid was prepared using plasma cleaner PDC-32G (HARRICK PLASMA, Ithaca, NY, USA) for $30 \mathrm{sec}$. TEM grids were loaded with $2 \mu \mathrm{L}$ from the diluted liquid culture, while excess liquid was dried using filter paper. 
Prepared grids were examined in FEI Tecnai T12 G2 TWIN transmission electron microscope operating at $120 \mathrm{kV}$.

\section{Swarming assay}

P. aeruginosa PA01 were grown overnight in LB-Miller medium. The next day, the bacteria were diluted 1:100 into fresh M9 medium and were grown to mid-log phase. The swarming plates were prepared using M9, solidified with $0.7 \%$ [wt/vol] Difco agar. Following bacteria refreshment, $2 \mu \mathrm{L}$ of the inoculums were placed in the middle of the plates enabling assessment of surface coverage after $24 \mathrm{hrs}$ of growth at $37^{\circ} \mathrm{C}$.

\section{Static biofilm growth}

Round glass coverslips, $15 \mathrm{~mm}$ diameter (Ted Pella, Inc., Redding, CA, USA) were incubated with $\mathrm{HCl} 8 \%$ for $1 \mathrm{hr}$ at room temperature and underwent autoclave sterilization in advance to biofilm growth. WT (PAO1 gGFP) or KO (PAO1 gGFP $\Delta f l i C$ ) were refreshed by taking $30 \mu \mathrm{L}$ stationary grown culture into $3 \mathrm{~mL}$ LB-Miller for $3 \mathrm{hrs}$ at $37^{\circ} \mathrm{C}$ with agitation. Reaching an $\mathrm{OD}_{600}$ of $\sim 0.2,20 \mu \mathrm{L}$ of refreshed culture were taken into $2 \mathrm{~mL}$ of LB-Lenox in a 12-well plate's well. Treated glass cover slip was placed in the well as well. Plate was incubated at $37^{\circ} \mathrm{C}$ for $16 \mathrm{hrs}$. Wells surrounding sample, were filled with DDW to keep high moisture during growth.

\section{High-resolution scanning electron microscopy (HR-SEM)}

Following static biofilm growth, liquid was aspirated and the glass cover slip underwent fixation $(2.5 \%$ glutaraldehyde, $2 \%$ paraformaldehyde, $0.2 \mathrm{M}$ phosphate buffer) for $15 \mathrm{~min}$. Samples were washed twice with PBS, 10 min each time, and were dehydrated using increasing ethanol concentrations before an addition of hexamethyldisilazane/ethanol solution in increasing concentrations. Finally, all liquids were removed and samples were dried in a chemical hood. 
751 Fixed glasses were coated in Cr using Quorum Q150T-ES sputter and examined in Verios 460L

752 Thermo Fisher Scientific scanning electron microscope operating at $3.00 \mathrm{kV}$.

753

754

755

756

757

758

759

760

761

762

763

764

765

766

767

768

769

770

771

772

773

\section{Dual-beam HR-SEM}

For an inside look at a biofilm and not just outer-rim observations, the same glasses coated with $\mathrm{Cr}$ that were used for HR-SEM, were also analyzed with dual-beam HR-SEM. Areas meant for examination were locally coated with Pt and were sliced using focused ion beam. Biofilm's inside was examined in Helios G4 UC Thermo Fisher Scientific scanning electron microscope operating at $5.00 \mathrm{kV}$.

\section{Atomic force microscopy (AFM) imaging}

Following static biofilm growth, the well was washed twice with PBS and left to dry in a hood. The dry samples were imaged on a MFP-3D-Bio (Oxford Instruments Asylum Research, Santa Barbara, CA, USA) in AC-mode ("tapping mode") in air, using an AC240BSA probe (Olympus) at room temperature. Imaging rate was of $0.5 \mathrm{~Hz}$, with parameters of 512 scan lines and 1024 scan points.

\section{AFM nanoindentation}

Local stiffness modulus, $K$, of the two different biofilms was measured with nano-indentation experiments performed on a Luigs \& Neurann LTD AFM. The biofilm samples were grown on a clean glass coverslip as was mentioned before. The grown biofilm on top the coverslip was gently washed with PBS and semi-dried in open air. The indentation was performed with pyramidal silicon nitride cantilevers (V-shape MLCT, Bruker) with a measured mean spring constant of $0.01 \mathrm{~N} / \mathrm{m}$. The normal spring constants of the cantilevers were determined before each measurement using the equipartition theorem ${ }^{54}$. In a nano-indentation measurement, force- 
774

775

776

777

778

779

780

781

782

783

784

785

786

787

788

789

790

791

792

793

794

distance curves are collected by approaching the cantilever tip towards the surface of the biofilm sample at a rate of $400 \mathrm{~nm} / \mathrm{s}$ and indentation depth amplitude of $500 \mathrm{~nm}$. Corresponding to the compliance of the sample, the cantilever deflects proportionally to the compliance of the sample.

The curvature of this response region was fitted using the Sneddon $\operatorname{model}^{55,56}$, a contact mechanics model for a conical sharp probe (as the one used), to the force distance curves:

$$
F=\frac{K}{1-v^{2}}\left(\frac{2}{\pi}\right) \tan (\alpha) \delta^{2}
$$

where $v$ is Poisson ratio, taken as 0.5 (typical value for incompressible materials), $\delta$ is the indentation length coordinate, $\theta$ is the cone half angle (face angle) of the AFM probe, taken as the manufacturer's nominal value of $29.1^{\circ}$, and $K$ is the local stiffness modulus. The indentation was performed at random locations across the surface of each biofilm sample. The measurement sets were repeated three times for each biofilm sample, where each set was comprised of 100 force-distance traces. All measurements were carried out under PBS buffer (150 mM NaCl, 20 $\mathrm{mM}$ PBS, $\mathrm{pH}$ 7.2) at room temperature. All data were recorded and analyzed using custom software written in Igor Pro 6.37 (Wavematrics).

From 100 collected values of $K$ for each biofilm, we constructed their probability distributions, and fitted them with the relevant statistical model. The Freedman-Diaconis rule was used as criterion for setting the bin size of the distributions ${ }^{57}$. The stiffness distribution of the WT biofilm was fitted with a Gaussian distribution, $\phi(K)=\left(\sigma(2 \pi)^{1 / 2}\right)^{-1} \exp \left\{-1 / 2[(K-\mu) / \sigma]^{2}\right\}$, and the stiffness distribution of the $\Delta f l i C$ biofilm was fitted with the Gamma distribution, which is given by $\phi(K)=\left(\beta^{\alpha} / \Gamma(\alpha)\right) K^{\alpha-1} \mathrm{e}^{-\beta K}$. This latter is highly useful to describe the distribution of a random variable that does not normally distribute. The mean is calculated by $\mu$ and $\alpha / \beta$ (for the normal and Gamma distributions respectively) and the variance (from which the standard 
795 deviation is calculated by taking its root) by $\sigma^{2}$ and $\alpha / \beta^{2}$ (for the normal and Gamma distributions 796 respectively).

797

\section{Additional references for online methods}

799 48. Nguyen, D. P. et al. Genetic Encoding and Labeling of Aliphatic Azides and Alkynes in

Chemistry. J. Am. Chem. Soc. 131, 8720-8721 (2009).

803

49. Ketema, M. et al. The rod domain is not essential for the function of plectin in maintaining tissue integrity. Mol. Biol. Cell 26, 2402-2417 (2015).

804

50. Dunphy, G. et al. Non-canonical Activation of the DNA Sensing Adaptor STING by $745-760$ (2018).

807 51. Waterhouse, A. et al. SWISS-MODEL: homology modelling of protein structures and complexes. Nucleic Acids Res. 46, W296-W303 (2018).

52. Yaniv, K. et al. Functional marine metagenomic screening for anti-quorum sensing and anti-biofilm activity. Biofouling 33, 1-13 (2017).

811 53. Labun, K. et al. CHOPCHOP v3: Expanding the CRISPR web toolbox beyond genome editing. Nucleic Acids Res. 47, W171-W174 (2019).

813 54. Hutter, J. L. \& Bechhoefer, J. Calibration of atomic-force microscope tips. Rev. Sci. Instrum. 64, 1868-1873 (1993).

815 55. Sneddon, I. N. The relation between load and penetration in the axisymmetric boussinesq problem for a punch of arbitrary profile. Int. J. Eng. Sci. 3, 47-57 (1965).

817 56. Krieg, M. et al. Atomic force microscopy-based mechanobiology. Nat. Rev. Phys. 1, 41- 
bioRxiv preprint doi: https://doi.org/10.1101/2020.08.26.267963; this version posted August 26, 2020. The copyright holder for this preprint (which was not certified by peer review) is the author/funder, who has granted bioRxiv a license to display the preprint in perpetuity. It is made available under aCC-BY-ND 4.0 International license.

819 57. Freedman, D. \& Diaconis, P. On the histogram as a density estimator:L2 theory. 
a

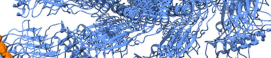

Le 30 (1)

bo

C

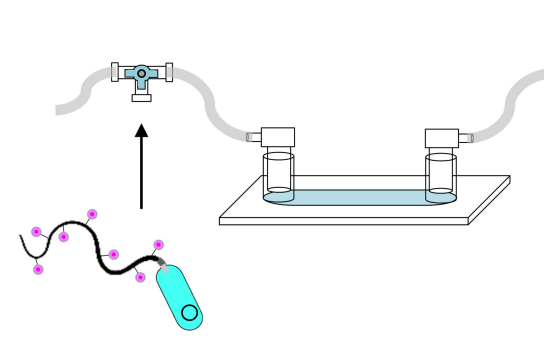

b

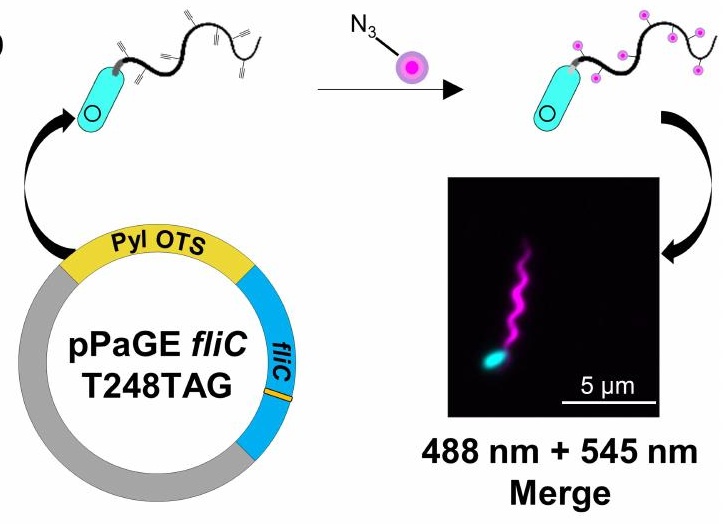

d
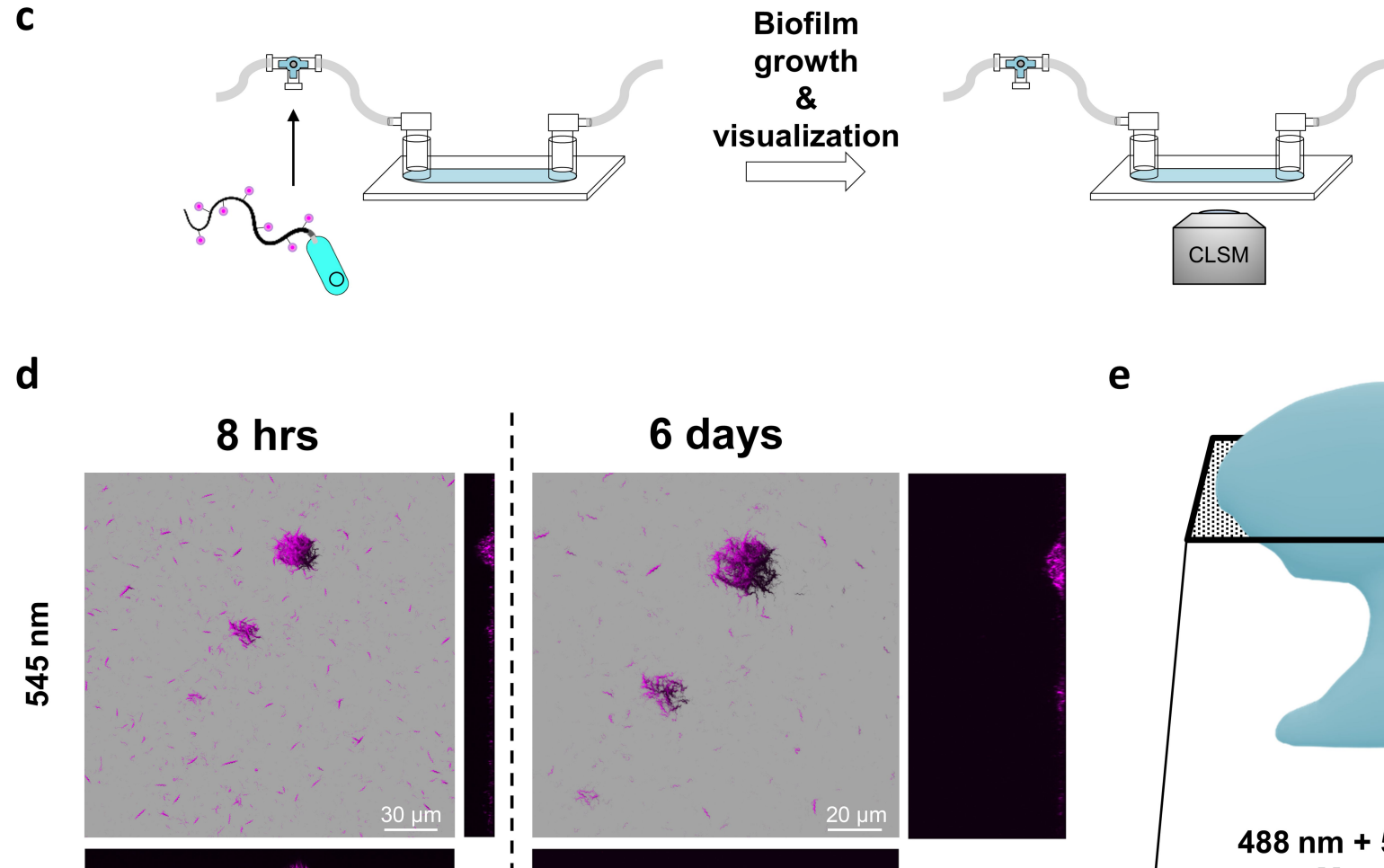

e
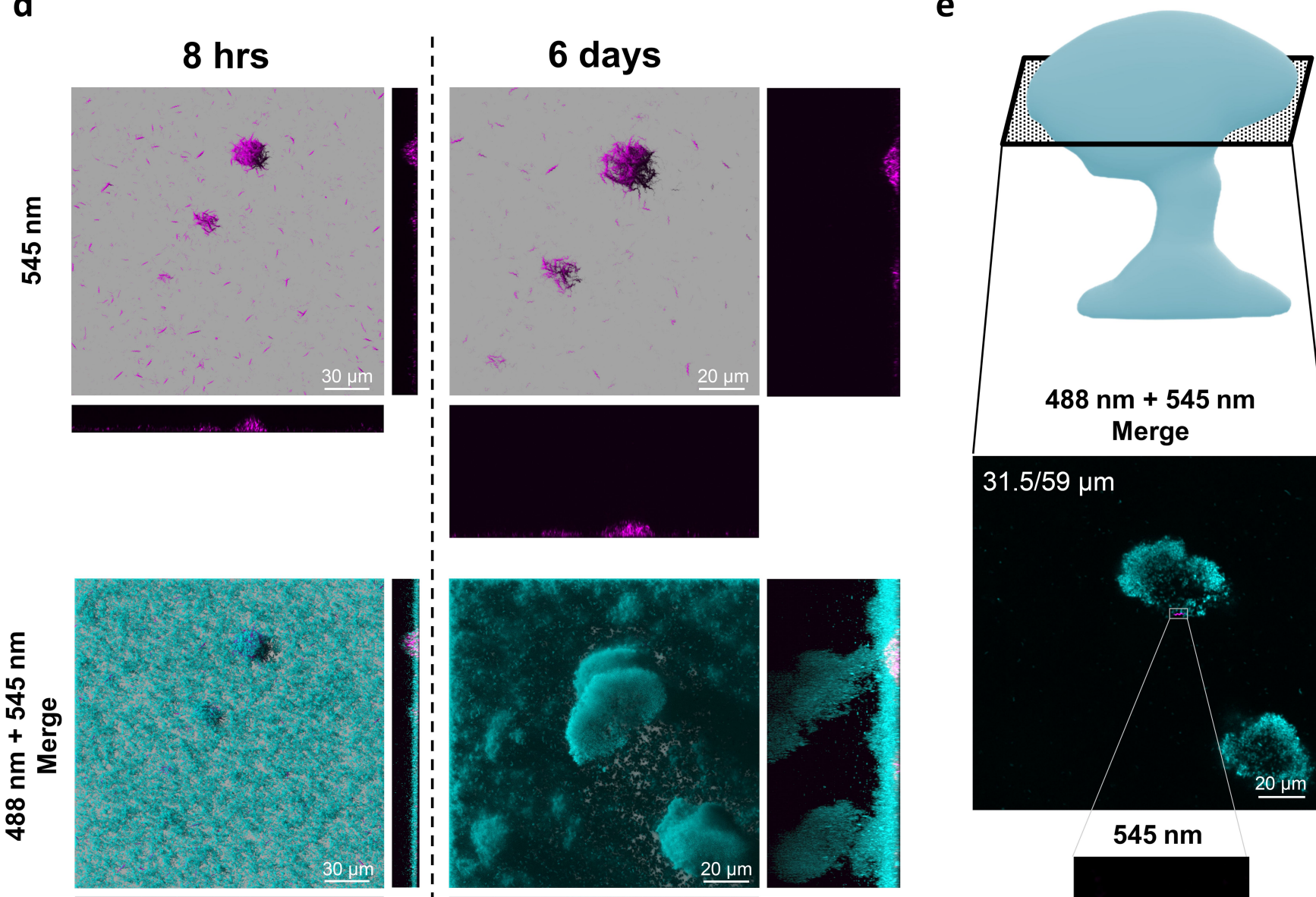

$31.5 / 59 \mu \mathrm{m}$

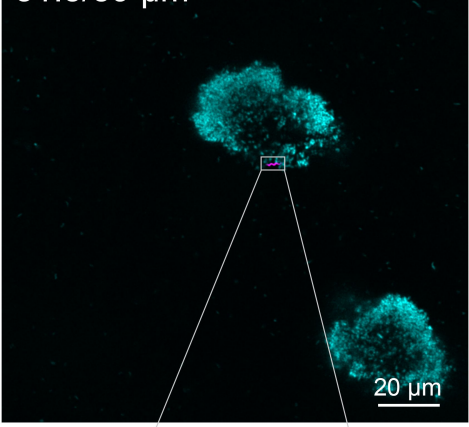

$545 \mathrm{~nm}$

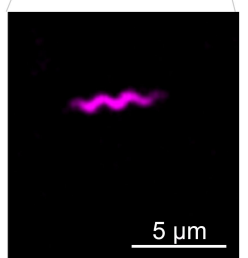



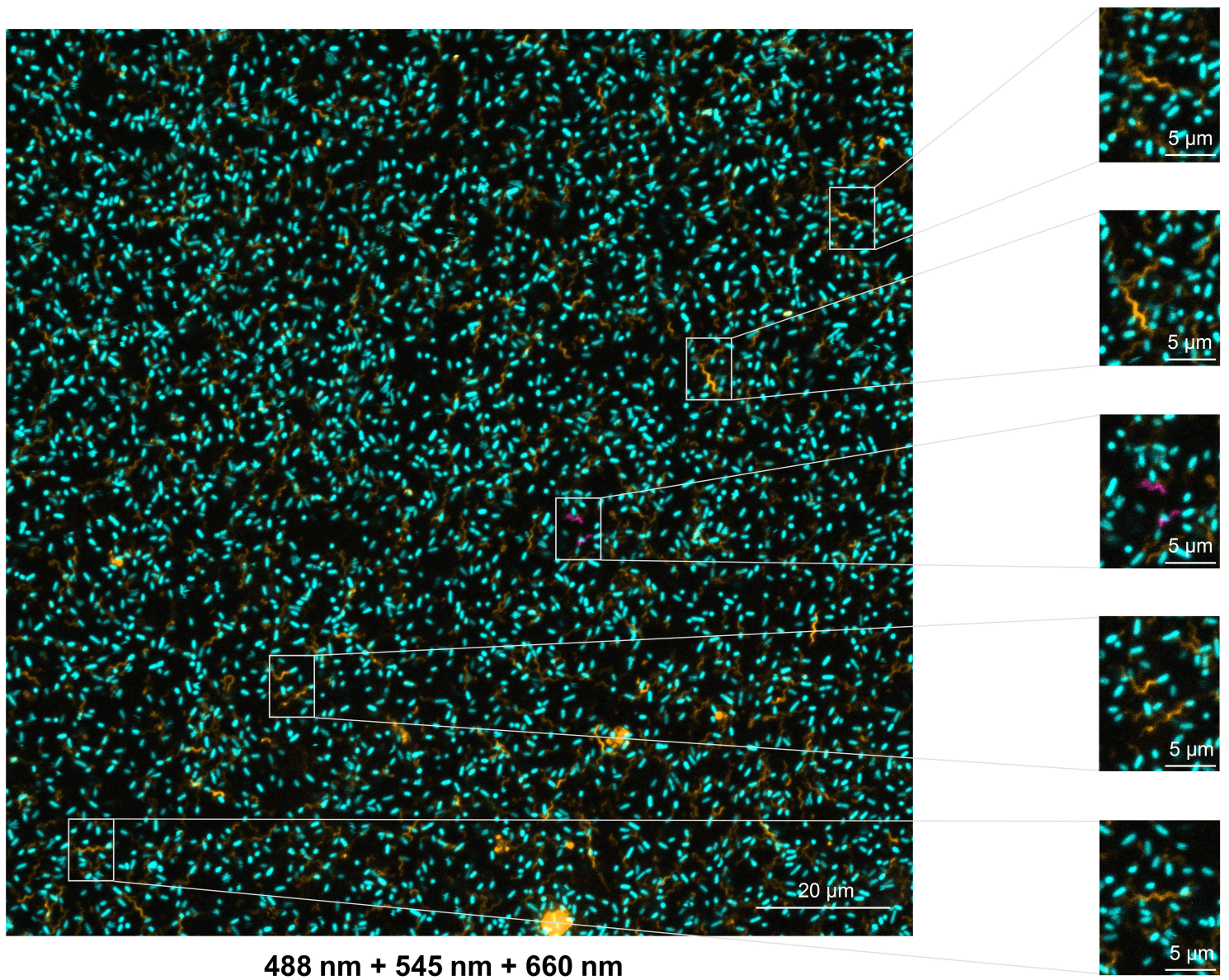
- Divided during biofilm growth (cyan+magenta)

3

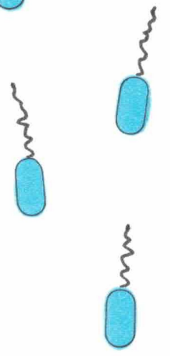
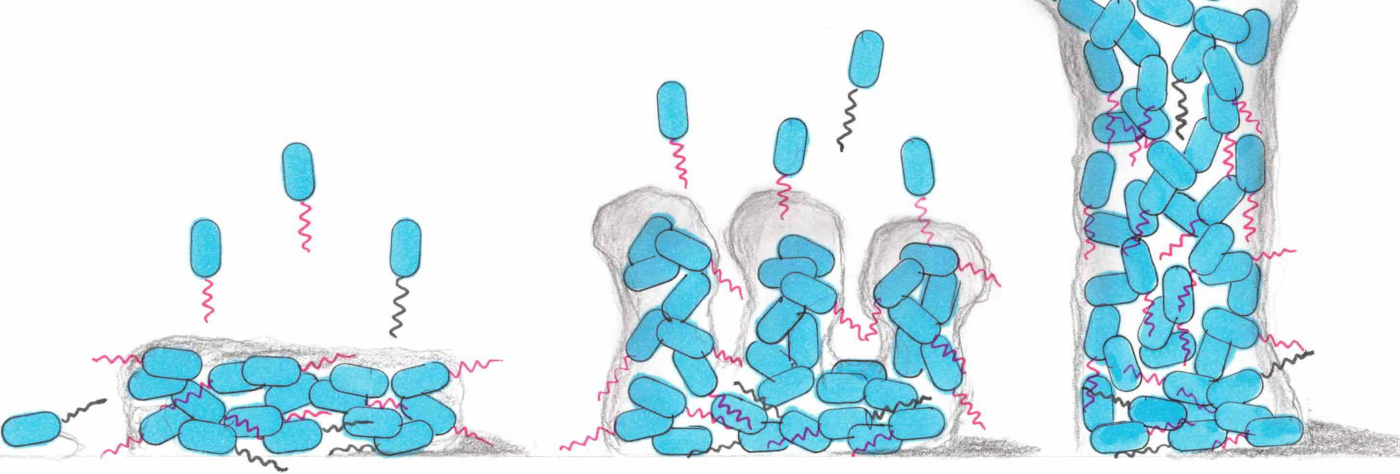


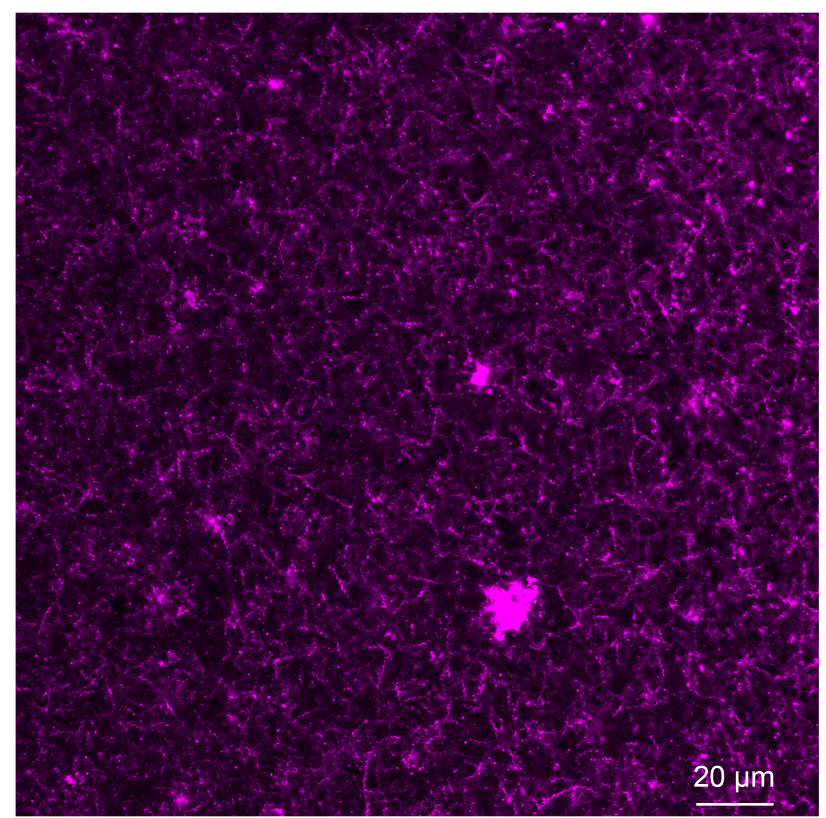

\section{$545 \mathrm{~nm}$}

C

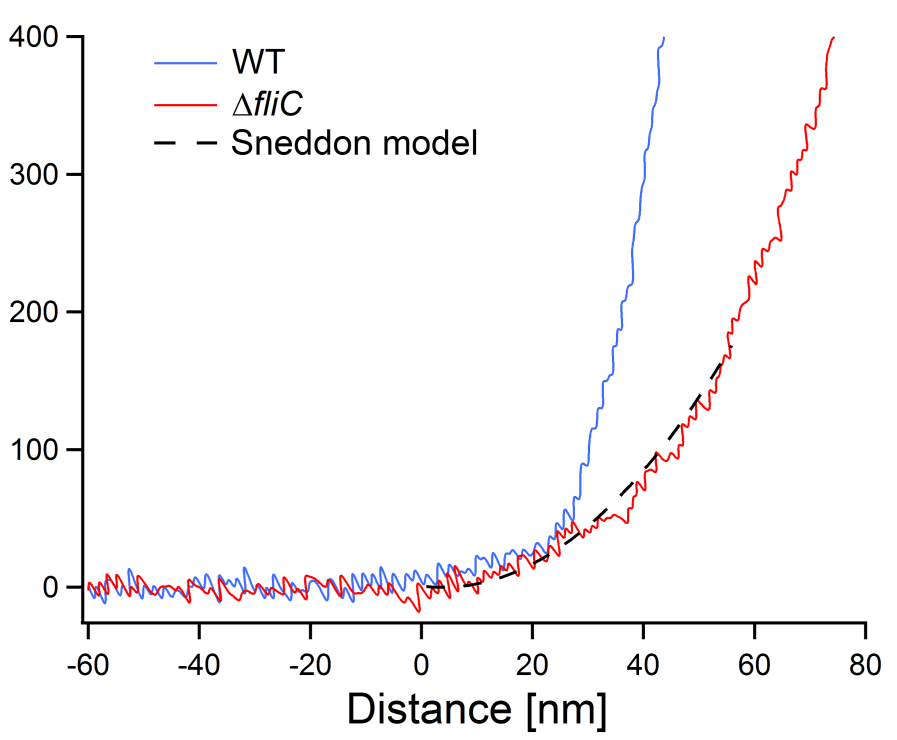

b

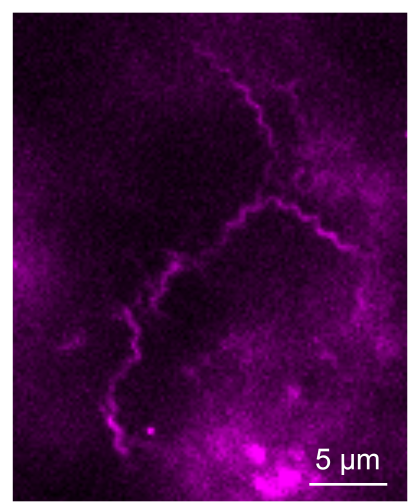

$545 \mathrm{~nm}$

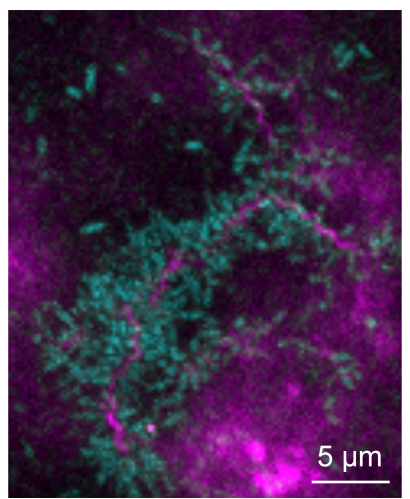

$488 \mathrm{~nm}+545 \mathrm{~nm}$ merge

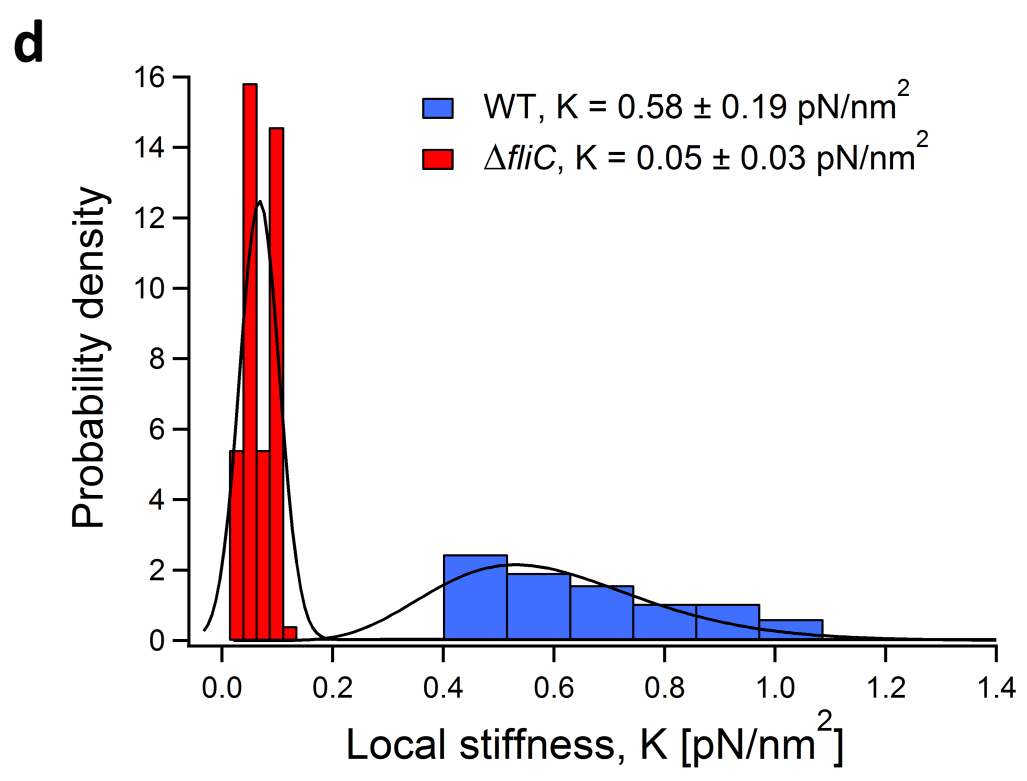

